\title{
Numerical Homogenization for Composite Materials Analysis. Comparison with Other Micro Mechanical Formulations
}

\author{
F. Otero ${ }^{\mathrm{a}, \mathrm{b}, *}, \mathrm{~S}$. Oller ${ }^{\mathrm{a}, \mathrm{b}}, \mathrm{X} \cdot$ Martínez $^{\mathrm{b}, \mathrm{c}}$, O. Salomón ${ }^{\mathrm{b}}$ \\ ${ }^{a}$ Departamento de Resistencia de Materiales y Estructuras en la Ingeniería, \\ ETSECCPB, Technical University of Catalonia, Spain \\ ${ }^{b}$ International Center for Numerical Methods in Engineering (CIMNE), Technical \\ University of Catalonia, Gran Capitá s/n, 08034 Barcelona, Spain \\ ${ }^{c}$ Departamento de Ciencia e Ingeniería Náutica, FNB, Technical University of \\ Catalonia, Pla de Palau 18, 08003 Barcelona, Spain
}

\begin{abstract}
This work presents a two-scale homogenization procedure to analyze three dimension composite structures by the finite element method. The theory implemented is compared with other micro-structural formulations: micro models and the serial-parallel mixing theory, in terms of result accuracy and computational cost. The comparison shows that for linear analysis, the homogenization proposed is an excellent alternative to the other formulations considered. Its computational cost is substantially lower than the one required by the micro-model and it is able to capture some micro-structural phenomena that it is not automatically recorded by the serial-parallel mixing theory. It will also be shown that the extension of the proposed theory to the non-linear range stills represents a challenge. The major limitation is its prohibitive computational cost because it requires solve the sub scale at each gauss point and each load step. However the comparison shows that this cost is in terms of CPU time but not in terms of memory. Based on the results obtained, it can be concluded that the homogenization method is an excellent alternative for the simulation of materials with complex micro structures. The method is also very promising for non linear simulations,

\footnotetext{
${ }^{*}$ Corresponding author

Email addresses: fotero@cimne.upc.edu (F. Otero), sergio.oller@upc.edu (S. Oller), x.martinez@upc.edu (X. Martínez), salomon@cimne.upc.edu (O. Salomón)
} 
when coupled with a threshold criteria to decide whether it is necessary to analyze the RVE or not.

Keywords:

Composites; Homogenization; Mechanical properties; Finite element method (FEM)

\section{Introduction}

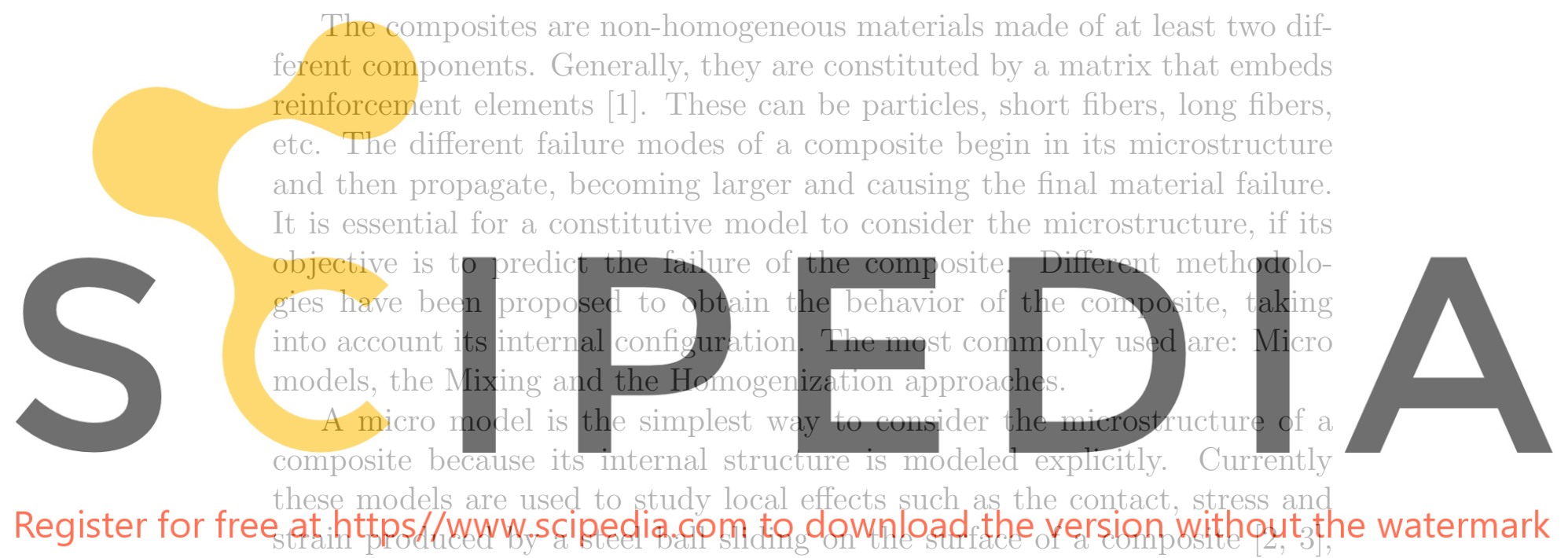

or to analyse of the propagation of a matrix crack from a debonded fiber [4]

or to study the effect of the interface, for a particle reinforced metal matrix composite, in the macro tensile stress/strain curve [5], etc. The problem with this kind of models is that its use is limited by its computational cost.

The mixing theory was proposed initially by Truesdell and Toupin [6]. Later, Green and Adkins [7] presented a general non-lineal constitutive equations. Ortiz and Popov [8, 9] proposed a general constitutive equations for unreinforced concrete idealized as a composite material. Then, the theory was modified by Oller et al. [10] and Neamtu et al. [11] introducing the serial-parallel concept. The mechanical characteristic of the composite are obtained using the properties of each component and taking into account its topological distribution. Oller generalizes the mixing theory to enable the resolution of any composite with reinforced matrix, without the limitation required by the compatibility equation [12]. The SP continuum approach proposed by Rastellini et al. [13] assumes the behavior components of the 
composite as parallel materials in the fibers alignment direction and as serial materials in the orthogonal direction. Recently, Otero et al. [14] presented the extension of the mixing theory for the study of matrices reinforced with nanotubes.

In the homogezation theory the problem is divided into two or more scales. One of the most relevant methods was proposed and developed by SanchezPalencia [15] and Bensoussan et al. [16], which is formulated in terms of asymptotic expansion theory. On the other hand, Suquet $[17,18]$ uses the method of averages to solve and extend the problem in two scales to the nonlinear case. Suquet laid the foundation of what today is known as first-order homogenization [19]. The deformation gradient tensor is used to solve the problem at the micro scale and then the macroscopic stress tensor is obtained using the averaging equation. Over time the method was extended to large deformations with arbitrary nonlinear material behaviour at the micro scale, thanks to the work of Moulinec [20], Miehe [21, 22, 23], Terada [24, 25]. Geers and Kouznetsova devdloped what is clled second-order homosenization 26.

27, 287; in this case the method uses the deformation gradient tensor and
its Lagrangian gradient to solve a boundary value problem at the micro
scale. The second-order approach allows solve the macro scale problem in
the presence of moderate localization, as macroscopic deformation gradients
(e.g. in bending) are passed to the RVE boundaries. To solve problems

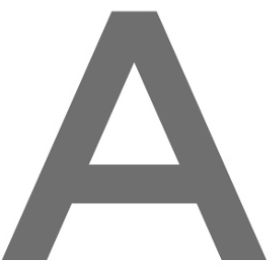
whe watermark

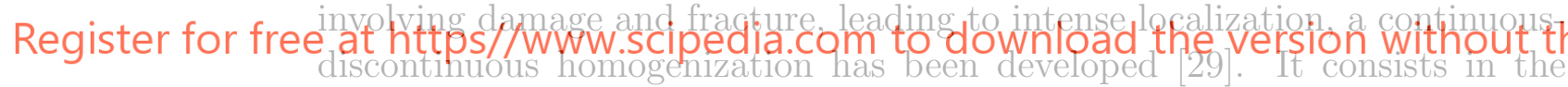
definition of a localization band at the macro scale, and using the deformation gradient tensor, in this band. In the last years, the homogenization has been extended to coupled thermo-mechanical problems [30], and to problems with cohesive zones [31], which can properly handle localization at the macroscopic scale.

This paper compares the results provides by these three numerical models (Micro models, Mixing and Homogenization approaches), looking into the strengths and weaknesses of each one of them. First, in the next section a brief description of Micro models and the Serial-Parallel theory, is done. The sections 3 and 4 describe the homogenization framework proposed and its implementation for three dimension composite structures. In the section 5 it is described the numerical model used in the section 6 to compare the results obtained with the different theories. The section 7 presents a computational cost comparative study considering all theories. Finally, the section 8 contains the conclusion of this work. 


\section{Numerical Models Used to Simulate the Microstructural's Be- havior}

This section briefly describes the numerical models that will be compared with the homogenization framework.

\subsection{Micro Models}

In these models, the constituent materials forming the composite are modeled explicitly. Therefore, the response of the composite arises naturally. Each single material is modeled with its own constitutive law. These models are very powerful because they do not need to take any hypothesis on the microstructural behavior. However, their biggest limitation is its computational cost and in most cases their use is not practical.

\subsection{Serial-Parallel Mixing Theory}
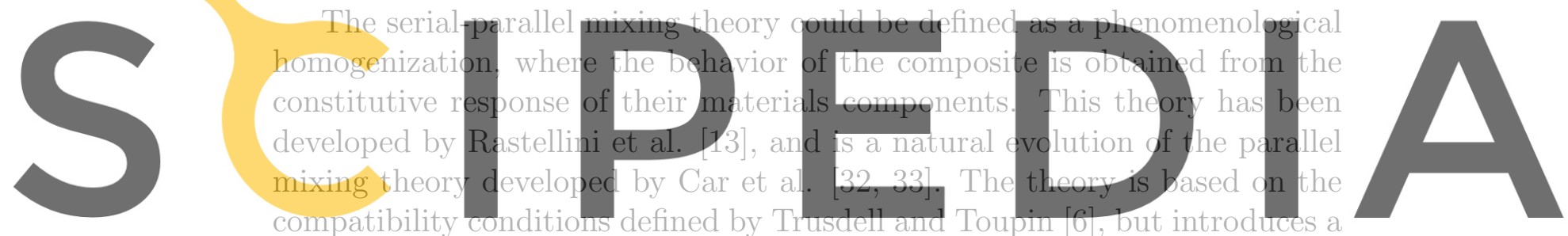

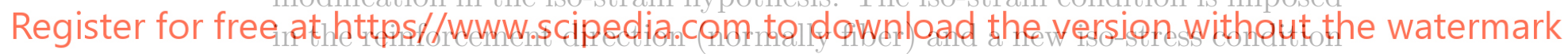

is imposed in the transversal directions. The theory is based on the following hypotheses:

1. The constituent materials of the composite are subjected to the same strain in the parallel (fiber) direction.

2. Constituent materials are subjected to the same stress in the serial direction.

3. The response of the composite material is directly related to the volume fractions of its constituent materials.

4. The phases in the composite are considered to be homogeneously ditributed.

5. The constituent materials are considered to be perfectly bonded.

Taking only two composite components, the equations that define the stress $(\sigma)$ equilibrium and setting up the strain $(\varepsilon)$ compatibility between the individual components follow the hypothesis previously described are: 
Parallel behavior:

$$
\begin{aligned}
& { }^{c} \varepsilon_{p}={ }^{m} \varepsilon_{p}={ }^{f} \varepsilon_{p} \\
& { }^{c} \sigma_{p}={ }^{m} k^{m} \sigma_{p}+{ }^{f} k^{f} \sigma_{p}
\end{aligned}
$$

Serial behavior:

$$
\begin{aligned}
& { }^{c} \varepsilon_{s}={ }^{m} k^{m} \varepsilon_{s}+{ }^{f} k^{f} \varepsilon_{s} \\
& { }^{c} \sigma_{s}={ }^{m} \sigma_{s}={ }^{f} \sigma_{s}
\end{aligned}
$$

where the superscripts $c, m$ and $f$ stand for composite, matrix and fiber, respectively and ${ }^{i} k$ is the volume-fraction coeffiecient of each constituent in the composite.

This theory can predict the linear and non linear behavior of structural elements made of composite materials. Composite materials that can be modelled are those formed of long fibers embedded in a matrix. The theory predicts the different behavior of the composite, depending on the load direction. The potential of this theory is to predict accurately the response of composites in the linear and non linear range (i.e. delamination failure)
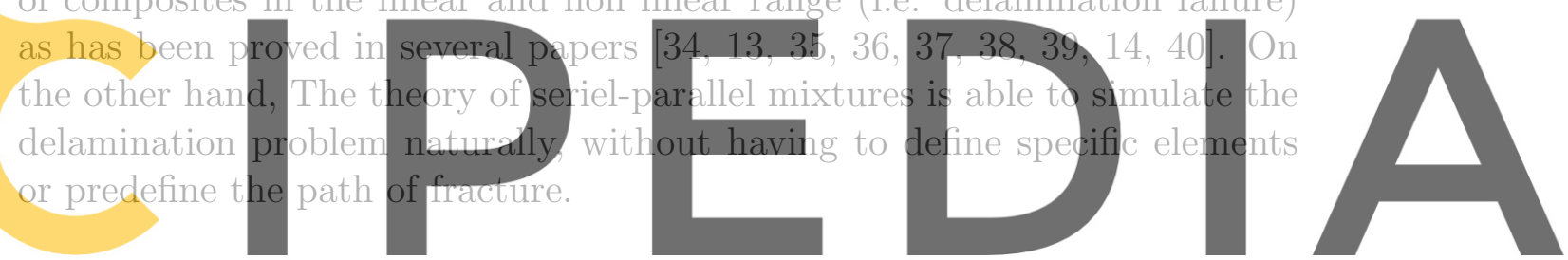

3. Multi-Scale Homogenization Model

Register for free at https//www.scipedia com to download the version without the watermark

edge from the theory proposed by Zalamea [41] and later extended by Oller

et al. [42] and by Badillo and Oller [43]. An extension to tri-dimensional framework of this theory is described in the following section.

The multi-scale homogenization is based on the use of an unit cell or representative volume element (RVE). The RVE definition corresponds to a microstructural subregion which is representative of the entire sub scale. The RVE is employed to determine the corresponding effective properties for the homogenized macro scale. For composites, it is assumed that it must contain a sufficient number of inclusions, which makes the effective moduli independent of assumed homogeneous forces or displacements on the RVE boundary.

In general, a multi-scale formulation that follows the first-order homogenization procedure can be identified as a "deformation driven" formulation. This mean that, with a deformation at the macro scale level, expressed by the strain tensor $\bar{E}$, the homogenized stress tensor $\bar{\sigma}$ and the homogenized 
constitutive tensor $\bar{C}$ can be determined at the sub scale level based on the interaction among the constituents at the RVE.

\subsection{Homogenized variables}

If the composite is periodic or quasi-periodic, its internal structure should be generated by repetition of their components. This property allows dividing the composite into a unit cell or RVEs in a way that each particle that conforms the mean is related to other particles that are distributed recurrently following a periodicity ratio. Therefore, any point inside the RVE has an equivalent point in each of neighboring RVE by periodicity. These points are known as periodic points and the link that connects each periodic point is known as a base vector of periodicity or periodicity vector.

When the unit cell suffers a change on its form, the periodic faces of the unit cell remain "parallel" by means of the periodicity vectors. This guarantees the compatibility of displacements in the upper scale, if not it would produce an overlap or form voids or gaps, in the composite. By the
principle of action and reaction. something similar occurs with the forces
generated on the boundary of the unit cell. The forces acting on the face
of an unit cell are transmitted with the same magnitude but in opposite
direction to the neighbor unit cell. This principle is called as a field of antiperiodic force.

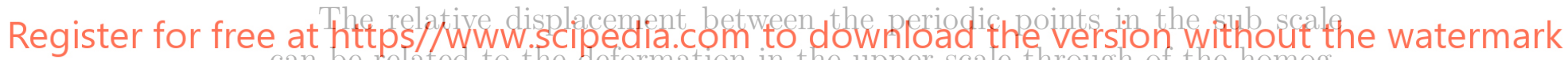

can be related to the deformation in the upper scale through of the homog-

enization of the strain and stress tensors, after this, it can be proposed and

solved the equation that dictates the balance of the composite structure.

\subsubsection{Homogenized strain tensor}

Consider a point $P$ inside a periodic composite material body $\Omega$, where a reference coordinate system $\overline{\mathbf{X}}$ is associated to the upper scale. Assuming that an amplification of the point $P$ could be represented by the unit cell domain $\Omega_{c}$, where a system of coordinates in the reference space $\mathbf{X}$ is defined, in a way that the material components of the composite can be perfectly identified. The space configuration at the unit cell domain $\Omega_{c}$ is associated with de periodicity vectors $\mathbf{D}$ in the space $\mathbf{X}$ the referential, meanwhile in the updated configuration system $\mathbf{x}$ the same space is associated to the vectors d as shown in the left scheme and the right scheme in Fig. 1, respectively.

The points $P_{0}, P_{1}, P_{2}$ and $P_{3}$, represent the periodic points in the reference coordinate system, therefore the periodicity vectors $\mathbf{D}$ are defined by the 


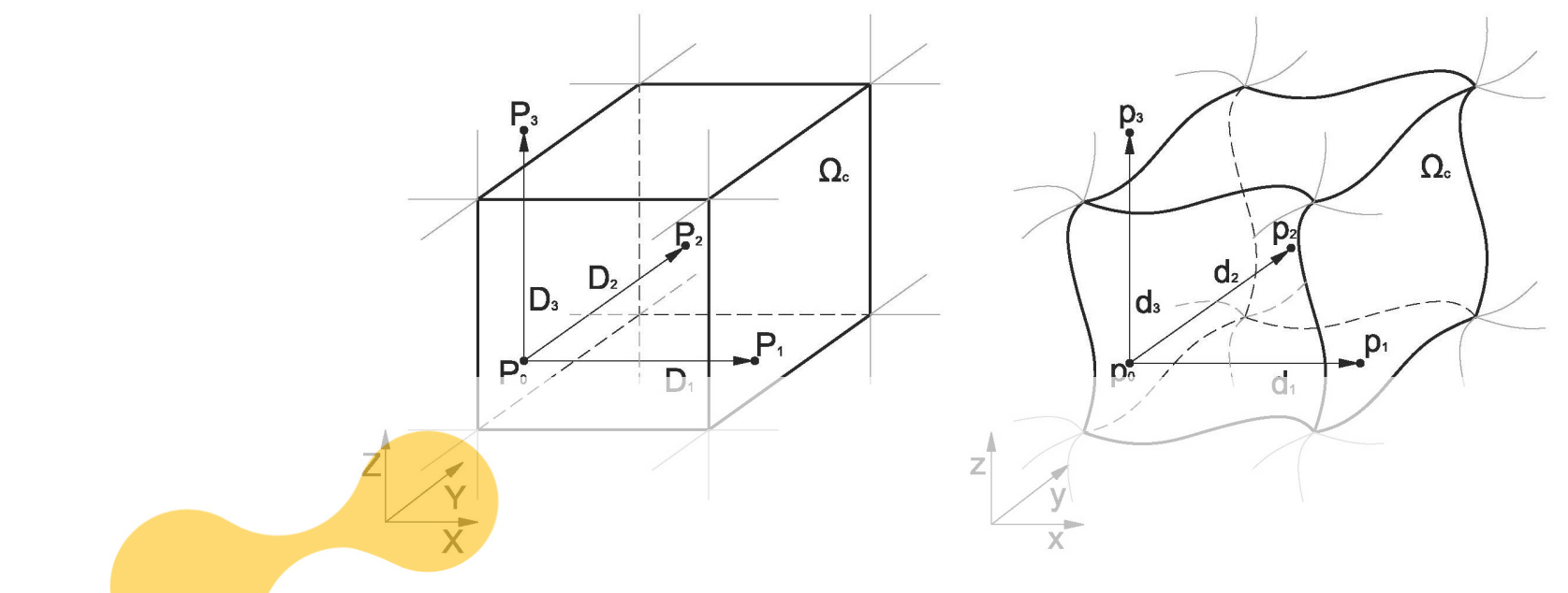

Figure 1: Schematic representation of the relation of the periodicity vectors in the referential and updated configurations.
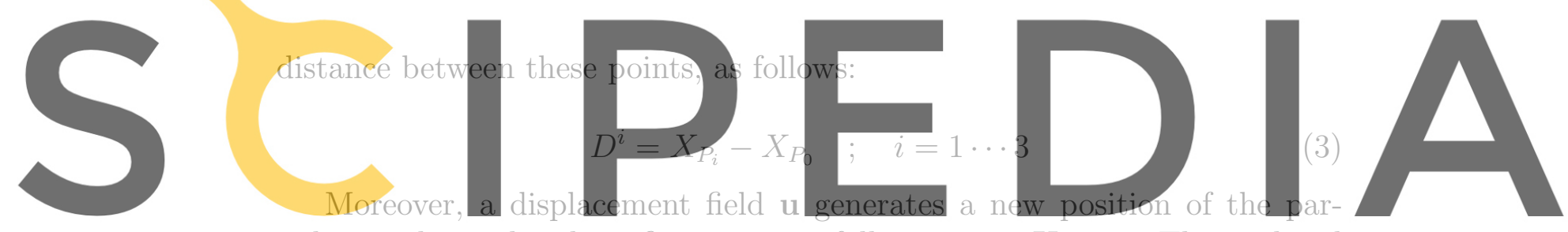

ticles at the updated configuration as follows: $\mathbf{x}=\mathbf{X}+\mathbf{u}$. The updated

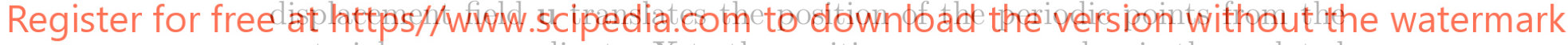

material space coordinates $\mathbf{X}$ to the position $p_{0}, p_{1}, p_{2}$ and $p_{3}$ in the updated

configuration $\mathbf{x}$. The hypothesis of local periodicity inposes that the mean is deformed but its variables maintain the local periodicity. Then, the unit cell is associated with a new periodicity vectors called the updated periodicity vector $\mathbf{d}$, defined as:

$$
d^{i}=x_{p_{i}}-x_{p_{0}}=D^{i}+u_{P_{i}}-u_{P_{0}} \quad ; \quad i=1 \cdots 3
$$

The transformation of the unit cell space is associated with the change of periodicity vectors. The parcial derivative of these vectors is:

$$
\frac{\partial \mathbf{d}}{\partial \mathbf{D}}=\frac{\partial\left(\mathbf{x}_{p}-\mathbf{x}_{p_{0}}\right)}{\partial\left(\mathbf{X}_{P}-\mathbf{X}_{P_{0}}\right)}
$$

Now, on the upper scale level, the periodicity vectors are infinitesimally small $(|\mathbf{D}| \rightarrow 0)$. Then the change over these vectors tends toward a limit: 


$$
\lim _{\mathbf{D} \rightarrow 0}\left[\frac{\partial \mathbf{d}}{\partial \mathbf{D}}\right]=\lim _{\mathbf{D} \rightarrow 0}\left[\frac{\partial\left(\mathbf{x}_{p}-\mathbf{x}_{p_{0}}\right)}{\partial\left(\mathbf{X}_{P}-\mathbf{X}_{P_{0}}\right)}\right]=\frac{\partial \overline{\mathbf{x}}}{\partial \overline{\mathbf{X}}}=\overline{\mathbf{F}}
$$

Therefore,

$$
\mathbf{d}=\overline{\mathbf{F}} \cdot \mathbf{D}
$$

where $\overline{\mathbf{F}}$ is the homogenized deformation gradient tensor. This change of scale allows determinig the homogenized strain tensor using concepts of the classical continuum mechanics. The square of the length of the updated periodicity vector is:

$$
|\mathrm{d}|^{2}=\mathrm{d}^{T} \cdot \mathrm{d}=\mathrm{D}^{T} \cdot \overline{\mathbf{F}}^{T} \cdot \overline{\mathrm{F}} \cdot \mathrm{D}
$$

And, the difference between the square of the length of the periodicity vectors in the updated and in the reference configurations is:
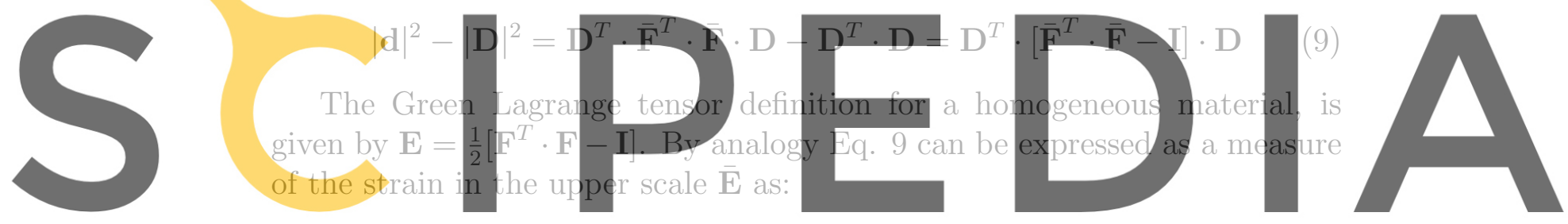

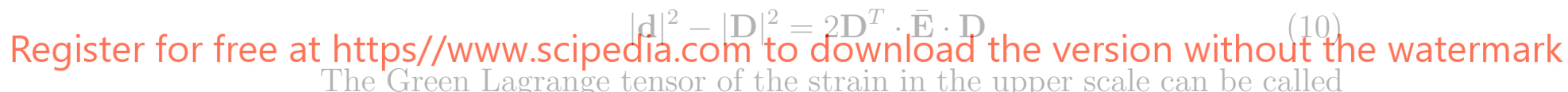
the homogenized strain tensor. This tensor is fully associated with the change of the periodicity vectors.

$$
\overline{\mathbf{E}}=\frac{1}{2}\left[\overline{\mathbf{F}}^{T} \cdot \overline{\mathbf{F}}-\mathbf{I}\right]
$$

The homogenized strain tensor quantifies the overall change of the unit cell's space under a periodic displacement field and it is independent of the patterns generated in the boundary of the cell. Moreover, it coincides with the classic equation of the averages theory proposed by Suquet [17].

$$
\overline{\mathbf{E}}=\langle\mathbf{E}(\mathbf{X}, t)\rangle_{\Omega_{c}}=\frac{1}{V_{c}} \int_{\Omega_{c}} \mathbf{E}(\mathbf{X}, t) d V_{c}
$$

where $\mathbf{E}(\mathbf{X}, t)$ is the sub scale strain tensor field at any instant $t$, defined over the domian of the unit cell $\Omega_{c}$ and $V_{c}$ is the volume contained in the cell's domain. 
Kinematically admissible displacement fields in the unit cell.

Considering the linear kinematic hypothesis, the strain tensor in the sub scale $\boldsymbol{\varepsilon}(\mathbf{x}, t)$ can be obtained as:

$$
\varepsilon(\mathbf{x}, t)=\nabla^{s} \mathbf{u}(\mathbf{x}, t)
$$

where $\nabla^{s} \mathbf{u}(\mathbf{x}, t)$ denotes the symmetric gradient of the sub scale displacement field $\mathbf{u}(\mathbf{x}, t)$ of the unit cell. Without loss generality it is possible to split $\mathrm{u}(\mathrm{x}, t)$ into in the following sum:

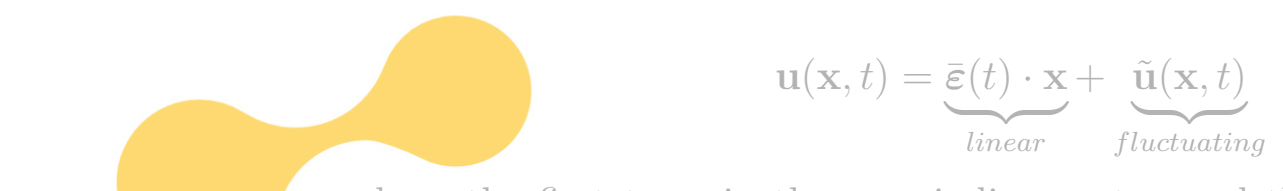

where the first term in the sum is linear at $\mathbf{x}$ and this also depends of the upper scale deformation tensor and $\tilde{\mathbf{u}}(\mathbf{x}, t)$ is a displacement fluctuation field
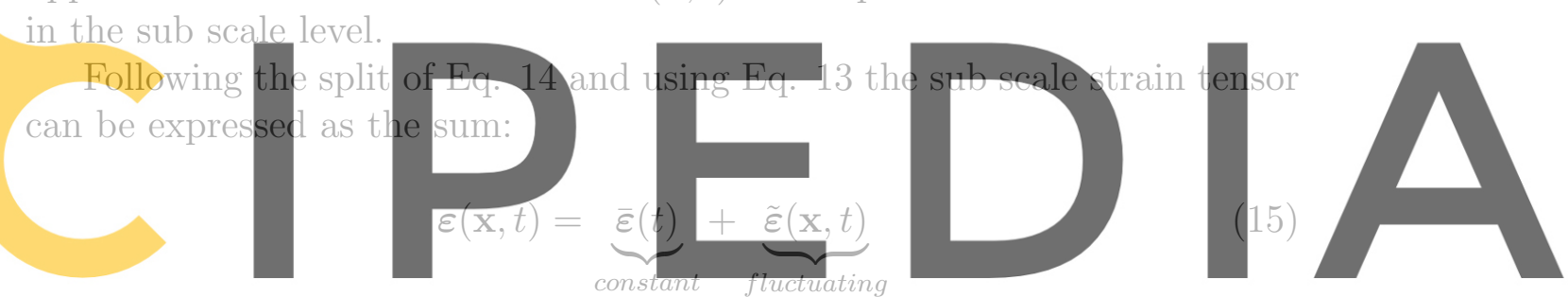

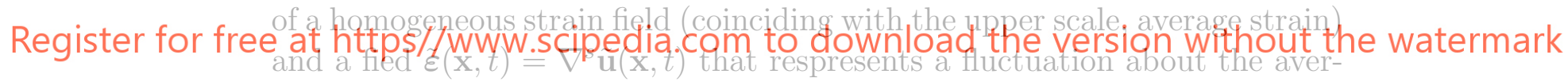
age.

On the othe hand, if replaced Eq. 13 into Eq. 12 and making use of Green's theorem, it can be established that the averaging relation of Eq. 12 is equivalent to the following constraint on the displacement field of the unit cell [44].

$$
\bar{\varepsilon}=\frac{1}{V_{c}} \int_{\Omega_{c}} \nabla^{s} \mathbf{u} d V_{c}=\frac{1}{V_{c}} \int_{\partial \Omega_{c}} \mathbf{u} \otimes^{s} \mathbf{n} d A_{c}
$$

where $\mathbf{n}$ denotes the outward unit normal field on $\partial \Omega_{c}$ and

$$
\mathbf{a} \otimes{ }^{s} \mathbf{b} \equiv \frac{1}{2}(\mathbf{a} \otimes \mathbf{b}+\mathbf{b} \otimes \mathbf{a})
$$

for any vectors a and b. Eq. 16 imposes constraints over the displacement field functions $\mathbf{u}(\mathbf{x}, t)$ in the sub escale level. Only the functions that satisfying this constraint are kinematically admissible, mathematically this is: 


$$
\mathbf{u} \in \mathcal{K}^{*} \quad ; \quad \mathcal{K}^{*}=\left\{\mathbf{u}, \text { sufficiently regular } \mid \int_{\partial \Omega_{c}} \mathbf{u} \otimes^{s} \mathbf{n} d A_{c}=\overline{\mathbf{E}} V_{c}\right\}
$$

where $\mathcal{K}^{*}$ is the minimally constrained set of kinematically admissible subescale displacements and with sufficiently regular meaning that the relevant functions have the sufficient degree of regularity so that all operations in which they are involved make sense.

Used Eq. 15, can easily be established that the constraint over the displacement field $\mathbf{u}(\mathbf{X}, t)$ are transferable to the displacement fluctuation field $\tilde{\mathbf{u}}(\mathbf{X}, t)$ as following:
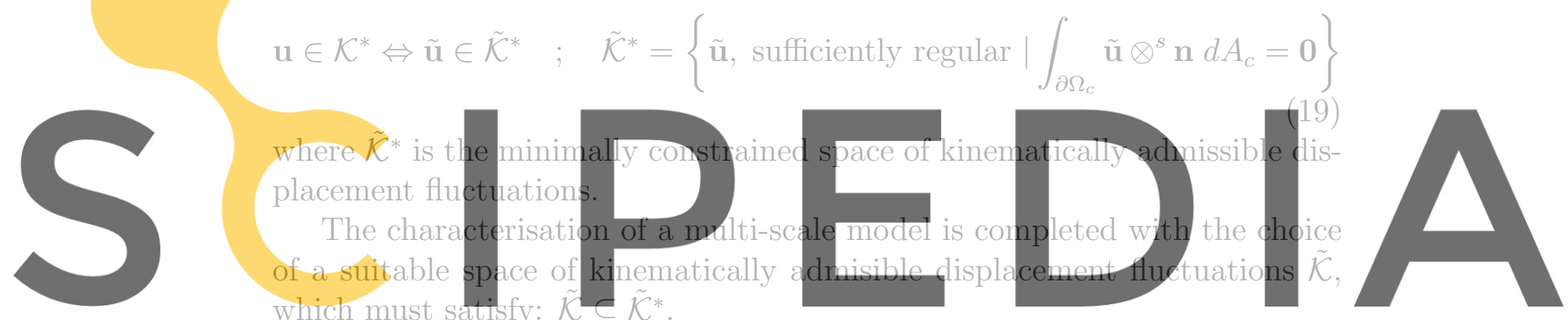

i) Taylor model (or zero fluctuations):

Register for free at https//www.scipedia.com to download the version without the watermark

$$
\tilde{\mathcal{K}}=\tilde{\mathcal{K}}^{\text {Taylor }} \equiv\left\{\tilde{\mathrm{u}}, \text { sufficiently regular } \mid \widetilde{\mathrm{u}}(\mathrm{x}, t)=0, \forall \mathrm{x} \in \Omega_{c}\right\}
$$

This model gives homogeneous deformation in the sub escale level.

ii) Linear boundary displacements (or zero boundary fluctuations):

$$
\tilde{\mathcal{K}}=\tilde{\mathcal{K}}^{\text {Lin }} \equiv\left\{\tilde{\mathbf{u}}, \text { sufficiently regular } \mid \tilde{\mathbf{u}}(\mathbf{x}, t)=\mathbf{0}, \forall \mathbf{x} \in \partial \Omega_{c}\right\}
$$

The deformation of the boundary of the unit cell for this class are fully prescribed as:

$$
\varepsilon(\mathbf{x}, t)=\bar{\varepsilon}(t) \forall \mathbf{x} \in \partial \Omega_{c}
$$

iii) Periodic boundary fluctuations:

This assumption is typically associated with the description of media with periodic microstructure [45]. The key kinematical constraint for this class of 
models is that the displacement fluctuatin must be periodic on the boundary of the unit cell. That is, for each pair $\left\{\mathbf{x}^{+}, \mathbf{x}^{-}\right\}$of boundary points it has:

$$
\tilde{\mathbf{u}}\left(\mathbf{x}^{+}, t\right)=\tilde{\mathbf{u}}\left(\mathbf{x}^{-}, t\right)
$$

Accordingly, the space $\tilde{\mathcal{K}}$ is defined as:

$$
\tilde{\mathcal{K}}=\tilde{\mathcal{K}}^{P e r} \equiv\left\{\tilde{\mathbf{u}}, \text { suff. reg. } \mid \tilde{\mathbf{u}}\left(\mathbf{x}^{+}, t\right)=\tilde{\mathbf{u}}\left(\mathbf{x}^{-}, t\right), \forall \text { pairs }\left\{\mathbf{x}^{+}, \mathbf{x}^{-}\right\} \in \partial \Omega_{c}\right\}
$$

iv) The minimally constrained (or uniform boundary traction):

$$
\tilde{\mathcal{K}} \equiv \tilde{\mathcal{K}}^{*}
$$

It has been shown by de Souza Neto and Feijóo [46] that a distribution of stress vector on the unit cell boundary as:

$$
\boldsymbol{\sigma}(\mathbf{x}, t) \cdot \mathbf{n}(\mathbf{x})=\overline{\boldsymbol{\sigma}}(t) \cdot \mathbf{n}(\mathbf{x}) \quad \forall \mathbf{x} \in \partial \Omega_{c}
$$

obtains the minimal kinematic constraint.

\subsubsection{Homogenized stress tensor}

The homogenized stress tensor $\overline{\boldsymbol{\sigma}}(\mathbf{x}, t)$ is defined as the average of the forces acting on the unit cell's sides:

$$
\overline{\boldsymbol{\sigma}}(\overline{\mathbf{x}}, t)=\frac{\int_{\partial \Omega_{c}} \mathbf{x} \otimes \boldsymbol{\sigma}(\mathbf{x}, t) \cdot \mathbf{n} d A_{c}}{\int_{\partial \Omega_{c}} \mathbf{x} \otimes \mathbf{n} d A_{c}}
$$

Thus, if the effect of the volume forces is disregarded $\left(\sigma_{i j, j}=0\right)$, and using the divergence theorem gives the following average theory equation:

$$
\overline{\boldsymbol{\sigma}}(\overline{\mathbf{x}}, t)=\frac{1}{V_{c}} \int_{\Omega_{c}} \boldsymbol{\sigma}(\mathbf{x}, t) d V_{c}
$$

The overall surface force $\overline{\mathbf{t}}(\hat{\mathbf{n}})$ is defined as the average of the forces on the boundary of the unit cell $\partial \Omega_{c}$ that is determined by the direction of the upper scale unitary vector $\hat{\mathbf{n}}$.

$$
\overline{\mathbf{t}}(\hat{\mathbf{n}})=\frac{1}{V_{c}} \int_{\Omega_{c}} \boldsymbol{\sigma}(\mathbf{x}, t) d V_{c} \cdot \hat{\mathbf{n}}=\overline{\boldsymbol{\sigma}} \cdot \hat{\mathbf{n}}
$$


Eq. 29 is a linear function that depends on the direction of $\hat{\mathbf{n}}$. The tensor $\overline{\boldsymbol{\sigma}}$ satisfies in the upper scale level the same requirements as the stress tensor $\boldsymbol{\sigma}$ for the case of homogeneous materials. Consequently, the tensor $\overline{\boldsymbol{\sigma}}$ hereinafter called as the homogenized stress tensor.

\subsubsection{Homogenized elastic constitutive tensor}

Considering that all the constituents are elastic and assuming that there is no relative displacement between these at the subscale, the homogenized elastic constitutive law relates the homogenized variables as:

$$
\overline{\boldsymbol{\sigma}}=\overline{\mathbf{C}}: \bar{\varepsilon}
$$

where $\overline{\mathbf{C}}$ is the tensor formed by the elastic constants of the homogenized composite, called homogenized elastic constitutive tensor.

The constitutive tensor is determined according to the theory of averages and the asymptotic expansion theory [16, 47, 48, 15, 19].

A possible procedure to obtain the homogenized constitutive tensor is by applying a homogenized $\bar{\varepsilon}$ strain on the cell domain in order to compute the homogenized stress $\overline{\boldsymbol{\sigma}}$ as it is done to calculate the tangent stiffness tensor in [35]. The homogenized strain can be applied in terms of small perturbations to the unit cell, and a system of equations with different perturbations in applied in each of the principal directions.

$$
\overline{\mathbf{C}}=\overline{\boldsymbol{\sigma}}: \bar{\varepsilon}^{-1}
$$

With this procedure the homogeneous constitutive tensor is obtained component by component.

\subsection{Linear-elastic homogenized formulation}

The behaviour of the upper scale is obtained by considering the cell as a structural unit and by considering the problem as a quasi-static problem expressed in small strains. The variables of the problem are established and the governing equations are formulated following this approach.

Equilibrium equations. The local stress field at the sub scale must satisfy an equilibrium equation inside the entire volume of the unit cell $\Omega_{c}$. The Cauchy equilibrium equation on the sub scale level may be written as

$$
\int_{\partial \Omega_{c}} \boldsymbol{\sigma} \cdot \mathbf{n} d A_{c}-\int_{\Omega_{c}} \rho \mathbf{b} d V_{c}=\mathbf{0}
$$


where $\mathbf{n}$ is a unit normal vector of the surface, $\rho$ is the mass, $\mathbf{b}$ is the force associated with the mass. The domain of the unit cell is very small from the upper scale point of view $\left(\Omega_{c} \rightarrow 0\right)$, Consequently, the value of the forces of volume and inertia are also small and tend to zero. Therefore Eq. 32 can be reformulated as:

$$
\lim _{\Omega_{c} \rightarrow 0}\left[\int_{\partial \Omega_{c}} \boldsymbol{\sigma} \cdot \mathbf{n} d A_{c}\right]=\mathbf{0} \quad ; \quad \lim _{\Omega_{c} \rightarrow 0}\left[\int_{\Omega_{c}} \rho \mathbf{b} d V_{c}\right]=\mathbf{0}
$$

The first integral of the above expressions indicates the concept of the balance in the microstruture, this is, the integral of the forces on the contour of the unit cell domain must be zero. Using the Divergence theorem in Eq. 33, the static equilibrium can be expressed as:

$$
\nabla \cdot \boldsymbol{\sigma}=\mathbf{0}
$$

Furthermore, if the entire domain of the periodic mean $\Omega$ is considered, and is represented as a homogeous material composed of a large number of alike unit cells, then it is possible to express the global balance equation as the integral of the balance equation on each of these cells:

$$
\int_{\partial \Omega}\left[\frac{1}{V_{c}} \int_{\Omega_{c}} \boldsymbol{\sigma}(\mathbf{x}, t) d V_{c}\right] \cdot \hat{\mathbf{n}} d A+\int_{\Omega}\left[\frac{1}{V_{c}} \int_{\Omega_{c}} \rho \mathbf{b} d V_{c}\right] d V=\mathbf{0}
$$

The body forces per unit volume have been considered because since on a upper scale level their magnitudes can be significant. This effect may be assumed as the average of the volume forces $\overline{\mathbf{b}}$ inside the unit cell as:

$$
\overline{\mathbf{b}}=\frac{1}{V_{c}} \int_{\Omega_{c}} \rho \mathbf{b} d V_{c}
$$

Using the divergence theorem of Eq. 35 can be rewritten as:

$$
\int_{\Omega} \nabla \cdot \overline{\boldsymbol{\sigma}} d V+\int_{\Omega} \overline{\mathbf{b}} d V=\mathbf{0}
$$

Eq. 37 is valid for any region $\Omega$, therefore it is also valid even when a very small domain is chosen, being the limit the unit cell. Then, the homogenized local equation of static equilibrium is obtained and it is expressed in the following way:

$$
\nabla \cdot \overline{\boldsymbol{\sigma}}+\overline{\mathbf{b}}=\mathbf{0}
$$




\subsubsection{Linear formulation at the upper scale}

A boundary value problem (BVP) is considered for the upper scale of a domain $\Omega$ with a periodic sub structure. The kinematics of the problem is related to a displacements field on the upper scale, which expresses the displacement of each particle of the domain $\Omega$. The BVP at the upper scale level must satisfy the following set of equations:

$$
\begin{array}{rr}
\frac{\partial \overline{\boldsymbol{\sigma}}(\overline{\mathbf{x}})}{\partial \overline{\mathbf{x}}}+\overline{\mathbf{b}}=\mathbf{0} & \text { equilibrium equation in } \Omega \\
\overline{\boldsymbol{\sigma}}(\overline{\mathbf{x}})=\frac{1}{V_{c}} \int_{\Omega_{c}} \boldsymbol{\sigma}(\mathbf{x}) d V_{c}=\overline{\mathbf{C}}: \overline{\boldsymbol{\varepsilon}}(\overline{\mathbf{x}}) & \text { constitutive equation in } \Omega \\
\mathbf{u}(\overline{\mathbf{x}})=\overline{\mathbf{u}}(\overline{\mathbf{x}}) & \text { displacements in } \partial \Omega_{u} \\
\overline{\boldsymbol{\sigma}}(\overline{\mathbf{x}}) \cdot \mathbf{n}=\overline{\mathbf{t}}(\overline{\mathbf{x}}) & \text { forces in } \partial \Omega_{t}
\end{array}
$$

where $\partial \Omega_{u}$ is the boundary in which the displacement is known (Dirichlet condition) and $\partial \Omega_{t}$ is the boundary where the forces are known (Neumann condition).

\subsubsection{Linear formulation at the sub scale}

To formulate the problem at the sub scale level it is necessary to establish special boundary conditions at the RVE domain $\Omega_{c}$. To ensure the compatibility of displacements at the upper scale level, a periodic boundary fluctuation displacements is used. Then, the relative displacement between the RVE boundary's periodic points or pair points $\left\{x^{+}, x^{-}\right\}$can be expressed as shown Eq. 45. Furthermore, by the principle of action and reaction, the sum of the forces generated on the RVE boundary $\partial \Omega_{c}$ (at the periodic points or pair points) must be zero. This condition ensures the periodicity of the field of internal forces in the upper scale level. Under these considerations, the problem is reduced to solve the following BVP in the domain of the RVE $\Omega_{c}$ :

$$
\begin{array}{rc}
\frac{\partial \boldsymbol{\sigma}(\mathbf{x})}{\partial \mathbf{x}}=\mathbf{0} & \text { equilibrium equation in } \Omega_{c} \\
\boldsymbol{\sigma}(\mathbf{x})=\mathbf{C}(\mathbf{x}): \boldsymbol{\varepsilon}(\mathbf{x}) & \text { constitutive equation in } \Omega_{c} \\
\mathbf{u}\left(x^{+}\right)-\mathbf{u}\left(x^{-}\right)=\overline{\boldsymbol{\varepsilon}}(\overline{\mathbf{x}}) \cdot \mathbf{D} & \text { periodic displacements in } \partial \Omega_{c} \\
\mathbf{t}\left(x^{+}\right)+\mathbf{t}\left(x^{-}\right)=\mathbf{0} & \text { periodic forces in } \partial \Omega_{c}
\end{array}
$$




\begin{tabular}{|l|l|l|l|l|l|l|l|l|}
\hline Nodes & a & b & c & d & e & f & g & h \\
\hline
\end{tabular}

Table 1: Periodic vertices nodes.

where $\mathbf{C}(\mathbf{x})$ is the constitutive tensor corresponding to the respective component at each point inside the domain.

\section{Numerical Implementation}

To solve the BVP in the sub scale it is necessary to verify the periodic displacements and periodic forces over RVE's boundary. Then, it is necessary to satisfy the Eqs. 45 and 46. If the BVP is solved by Finite Element Method (FEM) the unknowns are reduced to a finite degrees of freedom. The periodic boundary conditions are applied to the nodes on the RVE's boundary. A general RVE with hexagonal form it is shown in Fig. 2. The RVE has a coordinate system like the one shown in the left part of the Fig. 2, which origin is defined in node "a".

The restrictions of degrees of freedom on the boundary's domain can be accounted through several methods such as elimination of redundant unknowns, penalty methods and Lagrange multipliers [45]. The last two methods have the disadvantage that ill-conditioned stiffness matrix or increase the number of degrees of freedom of the problem. To avoid these two disadvantages, here is proposed solving the RVE by an elimination of redundant unknowns.

Eq. 45 shows the redundant unknowns of the problem. Using this equation it is possible identify master unknowns (the unknowns to resolve) and slave unknowns. Finally, the equation system to solve is reduced with Eq. 46.

The eight periodic vertices nodes will have a forced displacement which depends to the macro deformation (see left part of Fig. 2 and Table 1). If a structured Finite Element (FE) Mesh is used, it is possible identify easily master and slave nodes. The right part of Fig. 2 shows the chosen master nodes (named with a letter) and the slave nodes (named with a letter and number). The quantity of periodic nodes depends on the FE Mesh. Table 2 shows master nodes and the slave nodes located on the edges and surfaces of the RVE shown in Fig. 2.

Eq. 47 shows the forced displacements on the periodic vertice nodes, these only depend on the macro deformation, and RVE's dimensions. On 

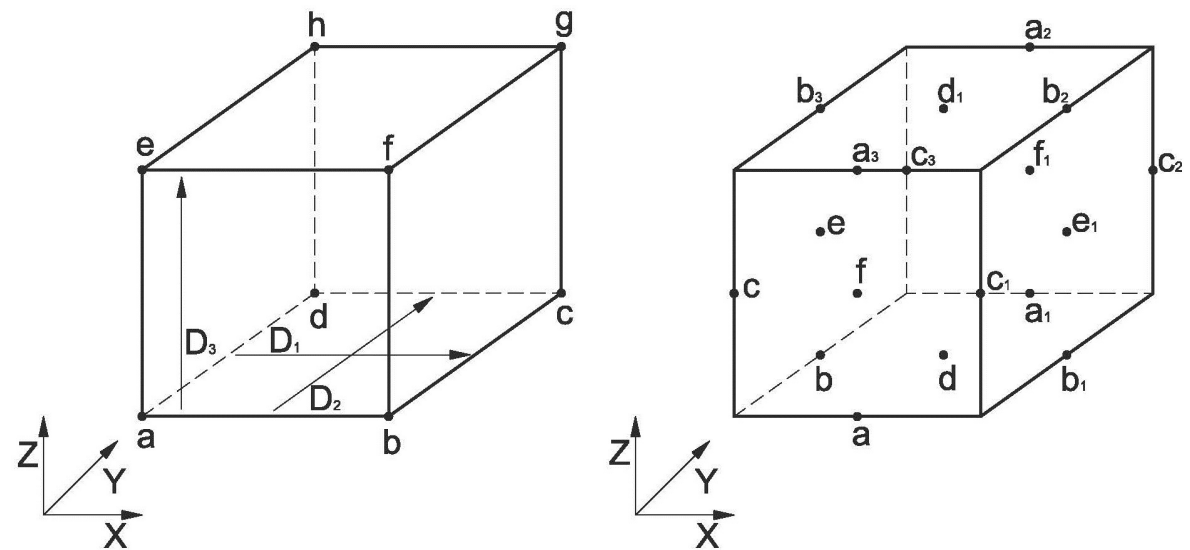

Figure 2: General hexagonal RVE.

\begin{tabular}{|c|c|c|c|c|c|c|}
\hline Master nodes & $\mathrm{a}$ & $\mathrm{b}$ & $\mathrm{c}$ & $\mathrm{d}$ & $\mathrm{e}$ & $\mathrm{f}$ \\
\hline Slave nodes & $\mathrm{a}_{1}, \mathrm{a}_{2}, \mathrm{a}_{3}$ & $\mathrm{~b}_{1}, \mathrm{~b}_{2}, \mathrm{~b}_{3}$ & $\mathrm{c}_{1}, \mathrm{c}_{2}, \mathrm{c}_{3}$ & $\mathrm{~d}_{1}$ & $\mathrm{e}_{1}$ & $\mathrm{f}_{1}$ \\
\hline
\end{tabular}

Table 2: Master and slave periodic nodes denomination.

the other hand, Eq. 48 shows the displacement relations between master and slave nodes. Finally, Eq. 49 gives the periodic forces relationship between the master and slave nodes.

$$
\begin{aligned}
& \mathrm{a}\left\{\begin{array} { l } 
{ \mathrm { a } _ { x } = 0 } \\
{ \mathrm { a } _ { y } = 0 } \\
{ \mathrm { a } _ { z } = 0 }
\end{array} \quad \mathrm { b } \left\{\begin{array} { l } 
{ \mathrm { b } _ { x } = \mathrm { E } _ { \mathrm { x } } \mathrm { D } _ { 1 } } \\
{ \mathrm { b } _ { y } = \mathrm { E } _ { \mathrm { xy } } / 2 \mathrm { D } _ { 1 } } \\
{ \mathrm { b } _ { z } = \mathrm { E } _ { \mathrm { xz } } / 2 \mathrm { D } _ { 1 } }
\end{array} \quad \mathrm { c } \left\{\begin{array}{l}
\mathrm{c}_{x}=\mathrm{E}_{\mathrm{x}} \mathrm{D}_{1}+\mathrm{E}_{\mathrm{xy}} / 2 \mathrm{D}_{2} \\
\mathrm{c}_{y}=\mathrm{E}_{\mathrm{y}} \mathrm{D}_{2}+\mathrm{E}_{\mathrm{xy}} / 2 \mathrm{D}_{1} \\
\mathrm{c}_{z}=\mathrm{E}_{\mathrm{xz}} / 2 \mathrm{D}_{1}+\mathrm{E}_{\mathrm{yz}} / 2 \mathrm{D}_{2}
\end{array}\right.\right.\right. \\
& \mathrm{d}\left\{\begin{array} { l } 
{ \mathrm { d } _ { x } = \mathrm { E } _ { \mathrm { xy } } / 2 \mathrm { D } _ { 2 } } \\
{ \mathrm { d } _ { y } = \mathrm { E } _ { \mathrm { y } } \mathrm { D } _ { 2 } } \\
{ \mathrm { d } _ { z } = \mathrm { E } _ { \mathrm { yz } } / 2 \mathrm { D } _ { 2 } }
\end{array} \quad \mathrm { e } \left\{\begin{array} { l } 
{ \mathrm { e } _ { x } = \mathrm { E } _ { \mathrm { xz } } / 2 \mathrm { D } _ { 3 } } \\
{ \mathrm { e } _ { y } = \mathrm { E } _ { \mathrm { yz } } / 2 \mathrm { D } _ { 3 } } \\
{ \mathrm { e } _ { z } = \mathrm { E } _ { \mathrm { z } } \mathrm { D } _ { 3 } }
\end{array} \quad \mathrm { f } \left\{\begin{array}{l}
\mathrm{f}_{x}=\mathrm{E}_{\mathrm{x}} \mathrm{D}_{1}+\mathrm{E}_{\mathrm{xz}} / 2 \mathrm{D}_{3} \\
\mathrm{f}_{y}=\mathrm{E}_{\mathrm{xy}} / 2 \mathrm{D}_{1}+\mathrm{E}_{\mathrm{yz}} / 2 \mathrm{D}_{3} \\
\mathrm{f}_{z}=\mathrm{E}_{\mathrm{z}} \mathrm{D}_{3}+\mathrm{E}_{\mathrm{xz}} / 2 \mathrm{D}_{1}
\end{array}\right.\right.\right. \\
& \mathrm{g}\left\{\begin{array} { l } 
{ \mathrm { g } _ { x } = \mathrm { E } _ { \mathrm { x } } \mathrm { D } _ { 1 } + \mathrm { E } _ { \mathrm { xy } } / 2 \mathrm { D } _ { 2 } + \mathrm { E } _ { \mathrm { xz } } / 2 \mathrm { D } _ { 3 } } \\
{ \mathrm { g } _ { y } = \mathrm { E } _ { \mathrm { y } } \mathrm { D } _ { 2 } + \mathrm { E } _ { \mathrm { xy } } / 2 \mathrm { D } _ { 1 } + \mathrm { E } _ { \mathrm { yz } } / 2 \mathrm { D } _ { 3 } } \\
{ \mathrm { g } _ { z } = \mathrm { E } _ { \mathrm { z } } \mathrm { D } _ { 3 } + \mathrm { E } _ { \mathrm { xz } } / 2 \mathrm { D } _ { 1 } + \mathrm { E } _ { \mathrm { yz } } / 2 \mathrm { D } _ { 2 } }
\end{array} \quad \mathrm { h } \left\{\begin{array}{l}
\mathrm{h}_{x}=\mathrm{E}_{\mathrm{xy}} / 2 \mathrm{D}_{2}+\mathrm{E}_{\mathrm{xz}} / 2 \mathrm{D}_{3} \\
\mathrm{~h}_{y}=\mathrm{E}_{\mathrm{y}} \mathrm{D}_{2}+\mathrm{E}_{\mathrm{yz}} / 2 \mathrm{D}_{3} \\
\mathrm{~h}_{z}=\mathrm{E}_{\mathrm{z}} \mathrm{D}_{3}+\mathrm{E}_{\mathrm{yz}} / 2 \mathrm{D}_{2}
\end{array}\right.\right.
\end{aligned}
$$




$$
\begin{aligned}
& \mathrm{a}_{1}\left\{\begin{array} { l } 
{ \mathrm { a } _ { 1 x } = \mathrm { a } _ { x } + \mathrm { E } _ { \mathrm { xy } } / 2 \mathrm { D } _ { 2 } } \\
{ \mathrm { a } _ { 1 y } = \mathrm { a } _ { y } + \mathrm { E } _ { \mathrm { y } } \mathrm { D } _ { 2 } } \\
{ \mathrm { a } _ { 1 z } = \mathrm { a } _ { z } + \mathrm { E } _ { \mathrm { yz } } / 2 \mathrm { D } _ { 2 } }
\end{array} \quad \mathrm { a } _ { 2 } \quad \left\{\begin{array}{l}
\mathrm{a}_{2 x}=\mathrm{a}_{x}+\mathrm{E}_{\mathrm{xy}} / 2 \mathrm{D}_{2}+\mathrm{E}_{\mathrm{xz}} / 2 \mathrm{D}_{3} \\
\mathrm{a}_{2 y}=\mathrm{a}_{y}+\mathrm{E}_{\mathrm{y}} \mathrm{D}_{2}+\mathrm{E}_{\mathrm{yz}} / 2 \mathrm{D}_{3} \\
\mathrm{a}_{2 z}=\mathrm{a}_{z}+\mathrm{E}_{\mathrm{z}} \mathrm{D}_{3}+\mathrm{E}_{\mathrm{yz}} / 2 \mathrm{D}_{2}
\end{array}\right.\right. \\
& \mathrm{a}_{3}\left\{\begin{array} { l } 
{ \mathrm { a } _ { 3 x } = \mathrm { a } _ { x } + \mathrm { E } _ { \mathrm { xz } } / 2 \mathrm { D } _ { 3 } } \\
{ \mathrm { a } _ { 3 y } = \mathrm { a } _ { y } + \mathrm { E } _ { \mathrm { yz } } / 2 \mathrm { D } _ { 3 } } \\
{ \mathrm { a } _ { 3 z } = \mathrm { a } _ { z } + \mathrm { E } _ { \mathrm { z } } \mathrm { D } _ { 3 } }
\end{array} \quad \mathrm { b } _ { 1 } \left\{\begin{array}{l}
\mathrm{b}_{1 x}=\mathrm{b}_{x}+\mathrm{E}_{\mathrm{x}} \mathrm{D}_{1} \\
\mathrm{~b}_{1 y}=\mathrm{b}_{y}+\mathrm{E}_{\mathrm{xy}} / 2 \mathrm{D}_{1} \\
\mathrm{~b}_{1 z}=\mathrm{b}_{z}+\mathrm{E}_{\mathrm{xz}} / 2 \mathrm{D}_{1}
\end{array}\right.\right. \\
& \mathrm{b}_{2}\left\{\begin{array} { l } 
{ \mathrm { b } _ { 2 x } = \mathrm { b } _ { x } + \mathrm { E } _ { \mathrm { x } } \mathrm { D } _ { 1 } + \mathrm { E } _ { \mathrm { xz } } / 2 \mathrm { D } _ { 3 } } \\
{ \mathrm { b } _ { 2 y } = \mathrm { b } _ { y } + \mathrm { E } _ { \mathrm { xy } } / 2 \mathrm { D } _ { 1 } + \mathrm { E } _ { \mathrm { yz } } / 2 \mathrm { D } _ { 3 } } \\
{ \mathrm { b } _ { 2 z } = \mathrm { b } _ { z } + \mathrm { E } _ { \mathrm { z } } \mathrm { D } _ { 3 } + \mathrm { E } _ { \mathrm { xz } } / 2 \mathrm { D } _ { 1 } }
\end{array} \quad \mathrm { b } _ { 3 } \quad \left\{\begin{array}{l}
\mathrm{b}_{3 x}=\mathrm{b}_{x}+\mathrm{E}_{\mathrm{xz}} / 2 \mathrm{D}_{3} \\
\mathrm{~b}_{3 y}=\mathrm{b}_{y}+\mathrm{E}_{\mathrm{yz}} / 2 \mathrm{D}_{3} \\
\mathrm{~b}_{3 z}=\mathrm{b}_{z}+\mathrm{E}_{\mathrm{z}} \mathrm{D}_{3}
\end{array}\right.\right. \\
& \mathrm{c}_{1}\left\{\begin{array} { l } 
{ \mathrm { c } _ { 1 x } = \mathrm { c } _ { x } + \mathrm { E } _ { \mathrm { x } } \mathrm { D } _ { 1 } } \\
{ \mathrm { c } _ { 1 y } = \mathrm { c } _ { y } + \mathrm { E } _ { \mathrm { xy } } / 2 \mathrm { D } _ { 1 } } \\
{ \mathrm { c } _ { 1 z } = \mathrm { c } _ { z } + \mathrm { E } _ { \mathrm { xz } } / 2 \mathrm { D } _ { 1 } }
\end{array} \quad \mathrm { c } _ { 2 } \quad \left\{\begin{array}{l}
\mathrm{c}_{2 x}=\mathrm{c}_{x}+\mathrm{E}_{\mathrm{x}} \mathrm{D}_{1}+\mathrm{E}_{\mathrm{xy}} / 2 \mathrm{D}_{2} \\
\mathrm{c}_{2 y}=\mathrm{c}_{y}+\mathrm{E}_{\mathrm{y}} \mathrm{D}_{2}+\mathrm{E}_{\mathrm{xy}} / 2 \mathrm{D}_{1} \\
\mathrm{c}_{2 z}=\mathrm{c}_{z}+\mathrm{E}_{\mathrm{xz}} / 2 \mathrm{D}_{1}+\mathrm{E}_{\mathrm{yz}} / 2 \mathrm{D}_{2}
\end{array}\right.\right. \\
& \mathrm{c}_{3}\left\{\begin{array} { l } 
{ \mathrm { c } _ { 3 x } = \mathrm { c } _ { x } + \mathrm { E } _ { \mathrm { xy } } / 2 \mathrm { D } _ { 2 } } \\
{ \mathrm { c } _ { 3 y } = \mathrm { c } _ { y } + \mathrm { E } _ { \mathrm { y } } \mathrm { D } _ { 2 } } \\
{ \mathrm { c } _ { 3 z } = \mathrm { c } _ { z } + \mathrm { E } _ { \mathrm { yz } } / 2 \mathrm { D } _ { 2 } }
\end{array} \quad \mathrm { d } _ { 1 } \left\{\begin{array}{l}
\mathrm{d}_{1 x}=\mathrm{d}_{x}+\mathrm{E}_{\mathrm{xz}} / 2 \mathrm{D}_{3} \\
\mathrm{~d}_{1 y}=\mathrm{d}_{y}+\mathrm{E}_{\mathrm{yz}} / 2 \mathrm{D}_{3} \\
\mathrm{~d}_{1 z}=\mathrm{d}_{z}+\mathrm{E}_{\mathrm{z}} \mathrm{D}_{3}
\end{array}\right.\right. \\
& \mathrm{e}_{1}\left\{\begin{array} { l } 
{ \mathrm { e } _ { 1 x } = \mathrm { e } _ { x } + \mathrm { E } _ { \mathrm { x } } \mathrm { D } _ { 1 } } \\
{ \mathrm { e } _ { 1 y } = \mathrm { e } _ { y } + \mathrm { E } _ { \mathrm { xy } } / 2 \mathrm { D } _ { 1 } } \\
{ \mathrm { e } _ { 1 z } = \mathrm { e } _ { z } + \mathrm { E } _ { \mathrm { xz } } / 2 \mathrm { D } _ { 1 } }
\end{array} \quad \mathrm { f } _ { 1 } \left\{\begin{array}{l}
\mathrm{f}_{1 x}=\mathrm{f}_{x}+\mathrm{E}_{\mathrm{xy}} / 2 \mathrm{D}_{2} \\
\mathrm{f}_{1 y}=\mathrm{f}_{y}+\mathrm{E}_{\mathrm{y}} \mathrm{D}_{2} \\
\mathrm{f}_{1 z}=\mathrm{f}_{z}+\mathrm{E}_{\mathrm{yz}} / 2 \mathrm{D}_{2}
\end{array}\right.\right. \\
& t_{\mathrm{a} 1}+t_{\mathrm{a} 2}+t_{\mathrm{a} 3}+t_{\mathrm{a}}=0 \quad t_{\mathrm{b} 1}+t_{\mathrm{b} 2}+t_{\mathrm{b} 3}+t_{\mathrm{b}}=0 \\
& t_{\mathrm{c} 1}+t_{\mathrm{c} 2}+t_{\mathrm{c} 3}+t_{c}=0 \quad t_{\mathrm{d} 1}+t_{\mathrm{d}}=0 \quad t_{\mathrm{e} 1}+t_{\mathrm{e}}=0 \quad t_{\mathrm{f} 1}+t_{\mathrm{f}}=0
\end{aligned}
$$

Using FEM it is possible to express the BVP for the sub scale problem as a discrete system of equations, which can be rewritten as following:

$$
\mathbf{K} \cdot \mathbf{d}=\mathbf{F}
$$

where $\mathbf{K}$ is the global stiffness matrix, $\mathbf{d}$ is the nodal displacement vector and $\mathbf{F}$ is the force vector on the RVE's boundary domain which result from applying the forced displacement on the periodic vertices nodes (see Eq. 47). However, Eq. 50 must include the restrictions of the periodic displacements and forces (Eqs. 48 and 49) as explained in the preceding paragraphs. The AppendixA shows the elimination of the redundant unknowns proposed. 


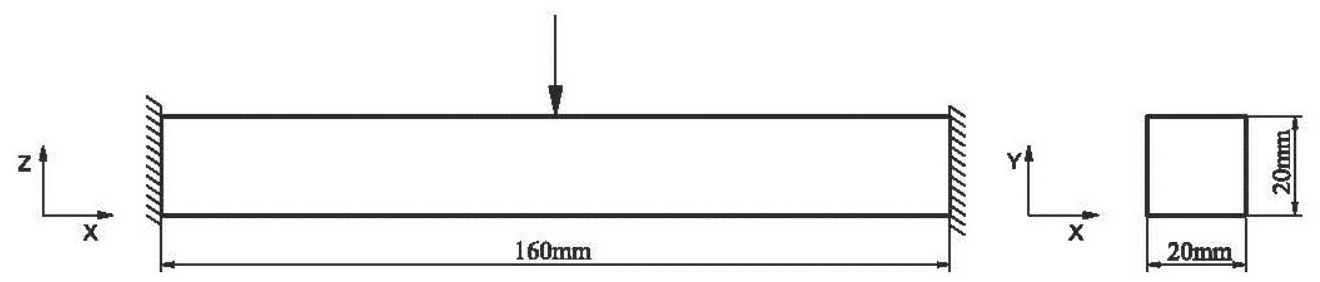

Figure 3: Geometric of the beam studied.

\section{Model Description}

A clamped beam with a vertical load at mid-span is the structure used to compare the theories presented previously: micro model, serial-parallel mixing theory and homogenization. Fig. 3 shows the beam's geometry, supports and loads. A macro numerical model will be used to simutale the behavior of the structure with the diffrent theories. In the special case of the homogenization theory another micro numerical model it is necessary. The micro numerical model will have the internal composite's structure.

\subsection{Macro and micro numerical model}

The macro FEM model used is the half of the beam because the symmetry of the structure (see Fig. 3). Fig. 4 shows the macro numerical model with one of the meshes used. The finite element used is a first order hexahedra element. In order to obtain the real behaviour of the structure with the FE model it is necessary to impose symmetric boundary conditions. The symmetry plane, the right face of Fig. 4, normal to $\mathrm{X}$-axis, and the $\mathrm{X}$ displacement is set to zero in this face. To simulate the fixed support, the nodes' movements in the left cross section are also restricted. The applied load is a $\mathrm{Z}$ direction fixed displacement of $-0.1 \mathrm{~mm}$ in the rigth cross section nodes (symmetry plane).

As said before, to simulate the RVE in the homogenization theory a micro numerical model is used. The RVE's geometry chosen is a cube with unit length sides. The finite element used is the same than the macro numerical model. The different RVE models that will be used are shown in the Figs. 5 and 8 . 


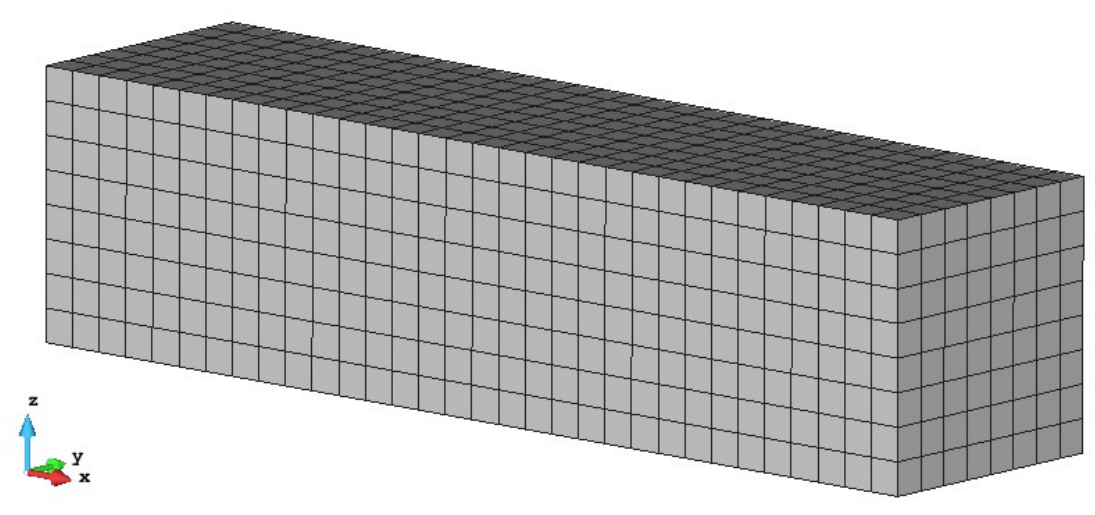

Figure 4: Beam numerical model

\begin{tabular}{lcccc}
\hline Simple mat. & Color ref. & $E(\mathrm{GPa})$ & $G(\mathrm{GPa})$ & $\nu$ \\
\hline Lamina 1 & Black & 210 & 80.76 & 0.3 \\
Lamina 2 & Grey & 3.5 & 1.46 & 0.2 \\
Lamina 3 & White & 3.5 & 0.146 & 0.2 \\
\hline
\end{tabular}

Table 3: Mechanical properties of the simple materials

\subsection{Simple materials and composite description}

The simple materials in all studied cases are isotropic elastic materials. The composite material is a laminate. And, the laminate consists of several layers of material 1, called lamina 1 henceforth, and a conbination of layers of material 2 (lamina 2) or material 3 (lamina 3). The volumetric participation of lamina 1 is always a $50 \%$.

Table 3 contains the mechanical properties of all the materials considered in the composite. In this table, $E$ is the Young's modulus, $G$ is the Shear modulus and $\nu$ is the poisson's ratio. The "Color ref." is the color used to represent the material in the RVEs, as it is shown in Figs. 5 and 8. The lamina 3 has the same properties as lamina 2, with the only difference of the shear moduluss, which is reduced by 10 . This is done to emulate the effect of a degradated lamina 2 . 


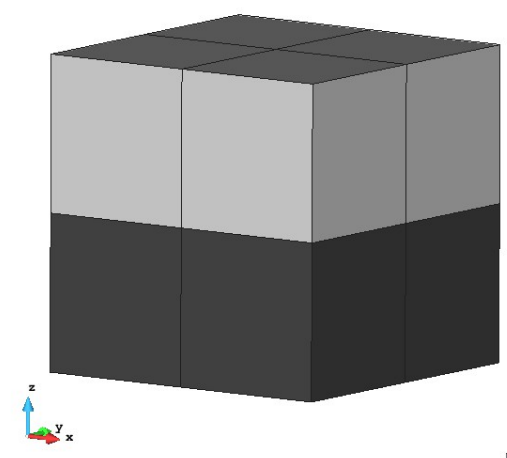

Figure 5: RVE used for the undamaged case.

\section{Comparison of the results obtained with the different formula- tions, for several material configurations}

In this section, several examples are presented to compare the behavior of the different theories. The result used to compare them is the reaction force, in $\mathrm{Z}$ direction, at the fixed support obtained for a fixed $\mathrm{Z}$ displacement applied at the symmetry plane (See Fig. 3) .

\subsection{Undamaged case}

The undamaged case is the first one used to compare all theories. In this case the laminate contains $50 \%$ of lamina 1 and $50 \%$ of lamina 2 , which properties are defined in Table 3.

The model using the SP mixing theory defines the composite material assuming that the parallel behavior is obtained in $\mathrm{X}$ and $\mathrm{Y}$ direction, while the rest of directions have a serial behavior. The homogenization theory uses a RVE made with 8 elements that also contains $50 \%$ of lamina 1 and $50 \%$ of lamina 2. The RVE is shown in the following Fig. 5. Finally, the micro-model is defined discretizing the different layers in which the laminate is divided.

A convergence analysis of SP and Homogenization theories has been made. The quantity of finite elements in the macro-model FE mesh has increased until the difference, between two consecutive results, is negligible. Fig. 6 presents the results obtained for the different meshes analysed. The micro-model used to compare the results obtained with the different theories 


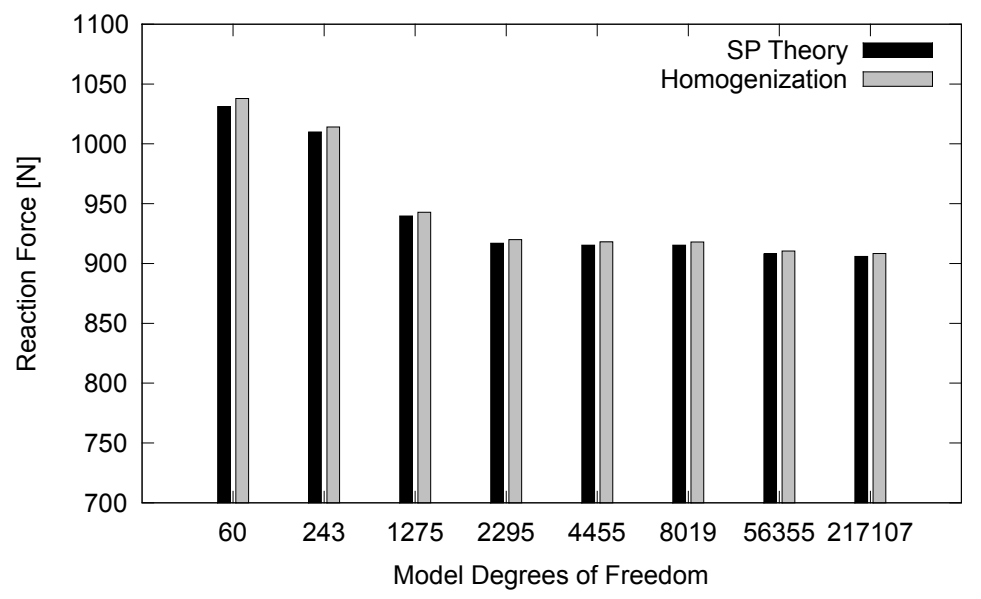

Figure 6: Convergence analysis results.

has been made with 196608 hexahedron elements, which results in 648999 degrees of freedom.

The reaction force obtained with the SP theory is $905.9 \mathrm{~N}$, with the homogenization is $908.3 \mathrm{~N}$ and with the micro-model is $919.0 \mathrm{~N}$. It can be concluded that the three theories provide almost the same result, as the difference between the reaction force value is lower than a $1 \%$ which is really good result. Besides, all theories allow knowing not only the global performance of the beam analyzed, but also the specific response of each lamina to the loads applied.

\subsection{Global damage case}

The objective for this example is to compare the responses obtained when one of the laminate materials suffers some sort of degradation. To analyze this problem, five different simulations have been performed in which the mechanical properties of one of the laminates is reduced. More specifically, the degradation is applied on the shear strength of lamina 2, which is reduced progressively until reaching the value of lamina 3 (see Table 3 ). Therefore, the new laminate consists $50 \%$ of lamina 1 and $50 \%$ of a new lamina that can be completely undamaged (properties of lamina 2) or with properties corresponding to $12.5 \%, 25 \%, 50 \%$ and $100 \%$ of damage (this last case, corresponds to lamina 3 ). The specific mechanical values considered are shown in Table 4. 


\begin{tabular}{lcccc}
\hline Property & $12.5 \%$ & $25 \%$ & $50 \%$ & $100 \%$ \\
\hline$G(\mathrm{GPa})$ & 1.295 & 1.131 & 0.803 & 0.146 \\
$E(\mathrm{GPa})$ & 3.5 & 3.5 & 3.5 & 3.5 \\
\hline
\end{tabular}

Table 4: Mechanical properties of the degradated Lamina 2

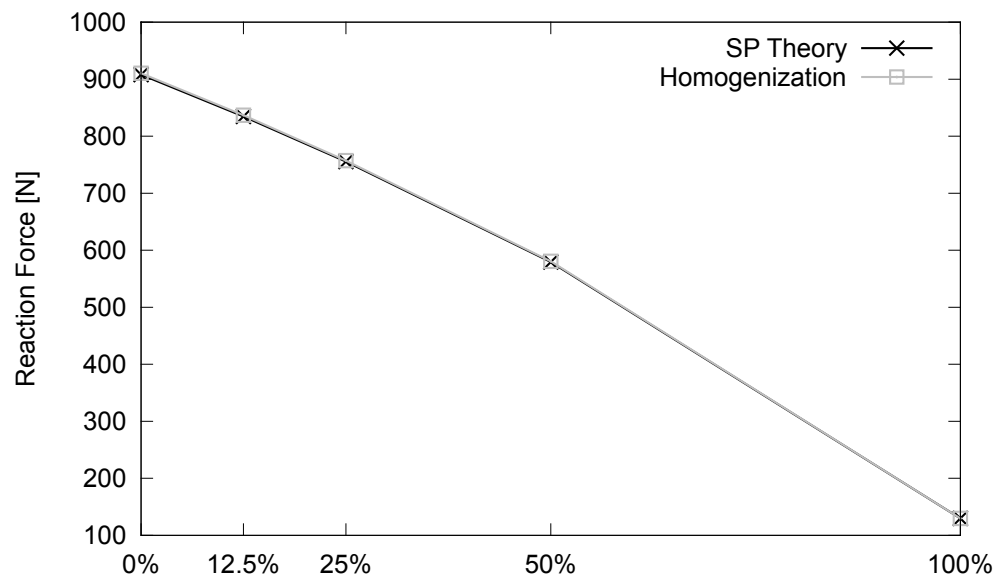

Figure 7: Reaction force obtained in the global damage case.

The composite is simulated with the SP mixing theory and with the homogenization theory, using the same material characterization and RVE that were used in the undamaged case. Fig. 7 shows the results obtained for the conducted simulations. This figure shows that, as it is expected, the results obtained for both theories are again exactly the same. The results obtained also show that as the shear stiffness of one of the layers is reduced, the global stiffness of the beam decreases. This effect can be understood as a delamination failure, as has been previously shown by Martinez et al. $[36,39]$. Results also show that the reduction of global stiffness of the beam is not linear with the reduction of the shear strength of one of the laminas, being larger as the layer stiffness gets smaller.

\subsection{Local damage case}

In this example, the objective is to compare the responses obtained when material damage takes place, not in all layers, but just in some of them. The composite considered has always $50 \%$ of lamina 1, and a $50 \%$ of lamina 2 (undamaged and damaged). It is assumed, like in the previous case, 

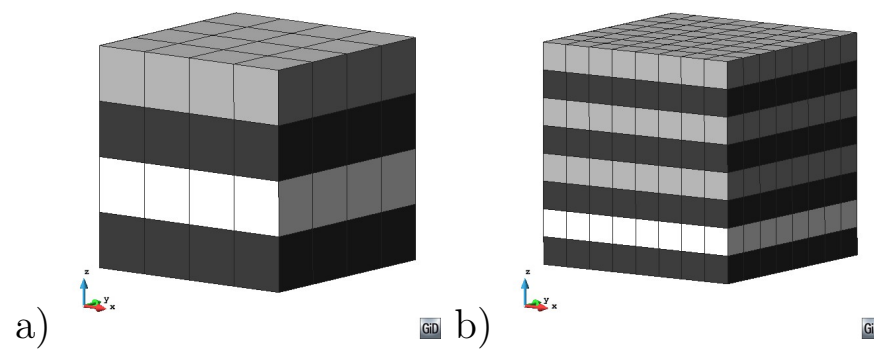

画 C)

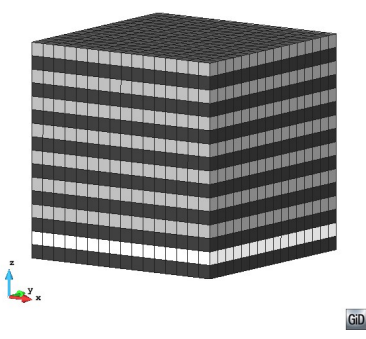

Figure 8: RVEs containing $50 \%, 25 \%$ and $12.5 \%$ of damaged layers.

that a totally damaged lamina 2 is numerally represented by the lamina 3 . The comparison is made for the cases in which there are $0 \%, 12.5 \%, 25 \%$, $50 \%$ and $100 \%$ of layers damaged. The simulation corresponding to $0 \%$ and $100 \%$ damaged have been already conducted in two previous simulations. The simulations corresponding to intermediate cases have been studied with the three methods being compared in this work: homogenization, SP and a micro-model.

For SP theory, the composite is obtained combining two different laminates with the SP formulation. One laminate has $50 \%$ of lamina 1 and $50 \%$ of lamina 2 and the other laminate has $50 \%$ of lamina 1 and $50 \%$ of lamina 3. The volume fraction of these laminates in the composite depend in the amount of layers assumed to be damaged. For the homogenization theory, the amount of layers damaged is represented with the RVE. Fig. 8 shows the RVEs considered to account for $50 \%, 25 \%$ and $12.5 \%$ of damaged layers, respectively. In this figure, the darker elements correspond to lamina 1, the light-grey elements correspond to lamina 2 (undamaged) and the white elements correspond to lamina 3 (damaged). Finally, the micro-model has been simulated discretizing each one of the lamina of the beam.

Fig. 9 presents the results obtained with different simulations performed. This figure shows that for the extreme cases, this is for $0 \%$ or $100 \%$ of lamina 3 , the results obtained with differents theories are almost equal. However, the results obtained when there are some layers damaged do not have the same agreement, especially when comparing the results obtained with the SP theory with the ones obtained with the homogenization method or the micro-model. While the decrease in the resultant reaction force with the SP theory is equal to the one obtained in the case of considering global damage (Fig. 7), this decrease is substantially larger when considering the 


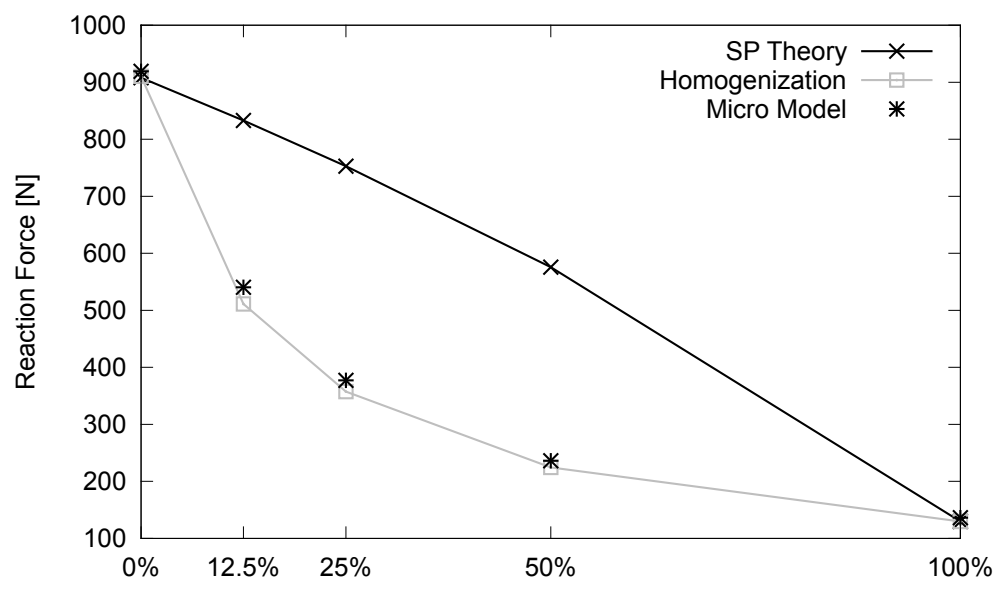

Figure 9: Reaction force obtained in the local damage case.

homogenization method or a micro-model. These two theories provide nearly the same results.

The explanation for the difference in the response obtained for the different models is obtained from the models themselves. The serial-parallel theory obtains the response of the composite assuming certain iso-stress and iso-strain boundary conditions that regularizes the response of the material if it is defined with several laminates. Therefore, the response of the structure and the result obtained is similar to the one obtained when this damage was present in the whole structure. On the other hand, with the homogenization and the micro-model theories, the damaged layer is discretized specifically and it is possible for the simulation to capture the dislocation that takes place, as it is shown in Fig. 10 for the three cases considered. This dislocation is the responsible for the drop of the stiffness and the fast decrement on the value of the reaction obtained.

\subsection{Local damage case in a localized region of the beam}

At the light of previous results one may think that the SP theory is not capable of representing delamination processes. In this example it is shown that under some circumstances this simulation is possible. Here, the beam has been simulated with two diffent laminates. The central band contains $50 \%$ of lamina 1 and $50 \%$ of lamina 3 (damaged); while the rest of the beam is simulated with $50 \%$ of lamina 1 and $50 \%$ of lamina 2. Fig. 11 shows the FE mesh of the beam macro-model. This example is simulated with the SP 
a)

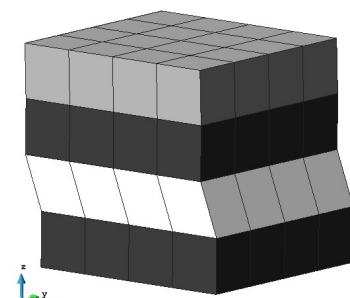

Gi. b)

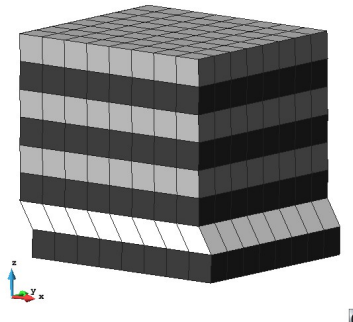

睲 c)

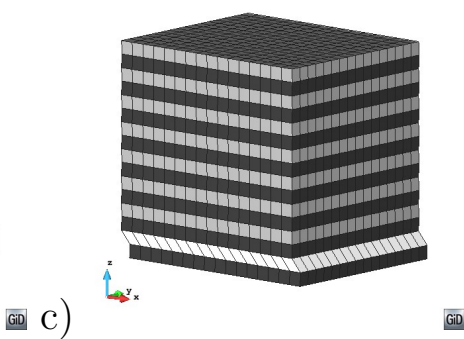

GiD

Figure 10: RVEs with $50 \%, 25 \%$ and $12.5 \%$ of damaged layers under load.

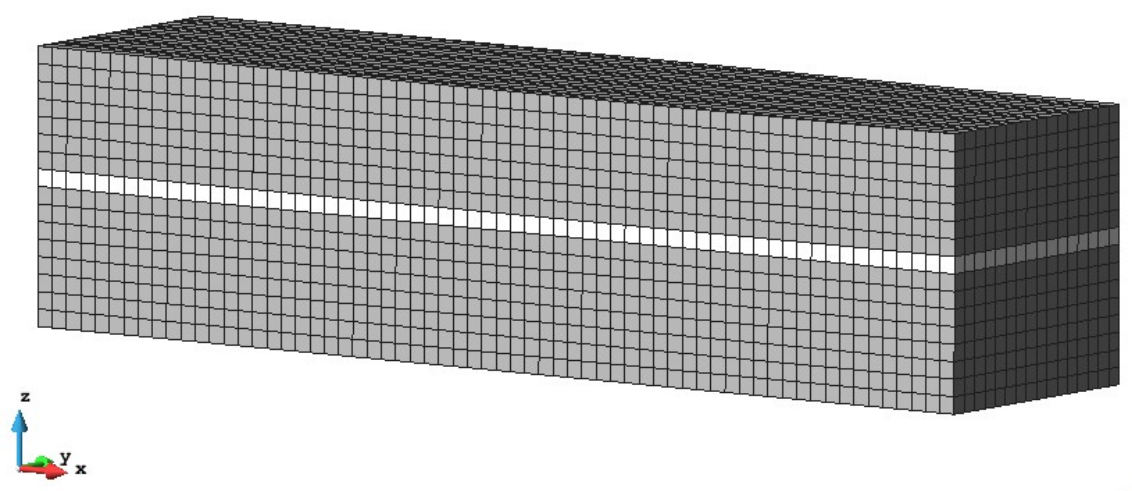

GiD

Figure 11: FE mesh of the macro-model of the beam with two laminates.

and the homogenization theory. The homogenization theory uses the RVE shown in Fig. 5.

In this case, the reaction force obtained is exactly the same for both, the SP and the homogenization models: $663.9 \mathrm{~N}$ and $666.4 \mathrm{~N}$, respectively. This example shows that the SP theory is capable of providing the same results as the homogenization theory when the response of the RVE fulfills the parameters in which is based the SP theory: iso-strain and iso-stress behavior. On the other hand, if the RVE does not fulfill this behavior (i.e. there is a dislocation in it), the SP theory is not capable of predicting accurately the material response, as it was shown in previous example. 


\section{Run Times and Memory Used}

One of the main drawbacks that has a homogenization formulation nowadays is its computational cost. Therefore, in order to know the performance of this formulation it is necessary not only to compare the results obtained with it, but also to compare the computational cost in terms of time and memory requirements.

To do these comparisons, in the case of the homogenization theory, two different strategies have been considered. In the first one, the mechanical properties of the composite (stiffness matrix) are calculated at the beginning of the analysis, and these porperties are used afterwards during the complete of the simulation. This case is named H-OneRVE. The other case corresponds to analyze the RVE each time that it is necessary to know the stress provided by the RVE for a given strain value. This case is named H-AllRVE. If the problem is linear, the results obtained in both cases are the same. However, in a non linear case, it is necessary to simulate the problem with an H-AllRVE strategy in order to capture properly the non linear response of the material.

Table 5 shows the computational times and memory required to conduct the simulations with a localized damage of a $50 \%$. The real time and cpu time are discriminated because part of the FE code used is in parallel. The results show that the CPU time in the Micro model and H-AllRVE are comparable. But, the CPU time of the H-OneRVE and SP theory are significantly better. Therefore, in terms of computational time, it is feasible to conduct a simulation with a homogenization theory, as well as with the SP theory. However, this simulation must be kept in the linear range. If the simulation is non linear, the H-AllRVE strategy must be used, which makes the SP theory the only feasible option in terms of computational time.

The main difference between the micro-model and the homogenized model is found in the memory requirements. While the computational time of the HAllRVE and the micro-model are equivalent, the amount of memory required by this last one is substantially larger (360 times larger). This difference

is found because the memory used is proportional to the FE mesh size of the numerical model and, while the micro-model has to solve a problem with a very small discretization, the homogenization theory only requires memory for the macro-problem and the RVE that is being solved. This difference makes unbearable solving large problems with micro-models and makes feasible using homogenization methods, even if the problem is in non linear range. 


\begin{tabular}{lcccc}
\hline Item & Micro Model & H-OneRVE & H-AllRVE & SP Theory \\
\hline Real Time [Min:Seg] & $6: 46$ & $0: 01$ & $2: 27$ & $0: 02$ \\
CPU Time [Min:Seg] & $8: 44$ & $0: 03$ & $9: 31$ & $0: 17$ \\
Memory [Mbytes] & 2690,00 & 7,45 & 7,45 & 15,82 \\
Reaction Force [N] & 236,09 & 224,69 & 224,69 & 576,68 \\
\hline
\end{tabular}

Table 5: Times and memory used to 50\% located damage case.

\section{Conclusion}

The two-scale homogenization framework proposed in this work, and described in detail in sections 3 and 4 , has been proved a competitive alternative to model three dimension composite structures. The elimination of redundant unknowns implemented to a cubic RVE is an efficiency opcion to satisfy the periodic boundary conditions. And, as the convergence analysis has shown, the method implemented has a good convergence behavior. Besides, for linear analysis, the comparison shows that the homogenization has many advantages over the other theories, such as micro-models or the SP theory, as it is capable of capturing complex responses of the material (such as dislocations) with an affordable computational cost.

The homogenization can represent accurately effects such as a local damaged lamina because the composite micro-structure is physically modeled in the RVE. The SP theory cannot account automatically for such effects, unless they are present in the whole finite element, as has been shown in the examples presented in subsection 6.2 and 6.4. However, the main advantage of the SP theory is that it is capable of conducting non linear analysis without increasing substantially the computational cost of the simulation.

The comparison of the computational cost presented by the different formulations has shown that, in terms of computational time, the cost of the H-OneRVE and the SP Theory is comparable, and it is substantially lower than the one required by a Micro Model or the H-AllRVE. The main difference between the H-AllRVE model and the micro-model is found in the memory required by the simulation, being the cost of this last one 360 times larger.

Therefore, this work has shown that the homogenization procedures are, nowadays, a real alternative for the linear analysis of composite structures. However, the extension of the homogenization methods to the non-linear range still represents a major challenge due to the computational cost in 
terms of CPU time. This is the main advantage of the SP mixing theory with respect to homogenization.

\section{Acknowledgements}

This work has been supported by the European Community under grant: NMP-2009-2.5-1 246067 M_RECT “Multiscale Reinforcement of Semi-crystalline Thermoplastic Sheets and Honeycombs" and by European Research Council through of Advanced Grant: ERC-2012-AdG 320815 COMP-DES-MAT "Advanced tools for computational design of engineering materials" and Amb el suport de la Universitat Politecnica de Catalunya (UPC).

\section{AppendixA. Elimination of the slave degrees of freedom}

Introducing the concept of master nodes and slave nodes into Eq. 50, the equation is rewritten as following:

$$
\left[\begin{array}{c|c|c}
K_{u u} & K_{u m} & K_{u s} \\
\hline K_{m u} & K_{m m} & K_{m s} \\
\hline K_{s u} & K_{s m} & K_{s s}
\end{array}\right]\left\{\begin{array}{l}
\frac{d_{u}}{d_{m}} \\
\hline d_{s}
\end{array}\right\}=\left\{\begin{array}{c}
\frac{F_{u}}{F_{m}} \\
\hline F_{s}
\end{array}\right\}
$$

where the subscripts $u, m$, and $s$ refer to the degrees of freedom of the internal nodes, master nodes and slave nodes, recpectively.

If the contribution of each type of degree of freedom is separated, this is:

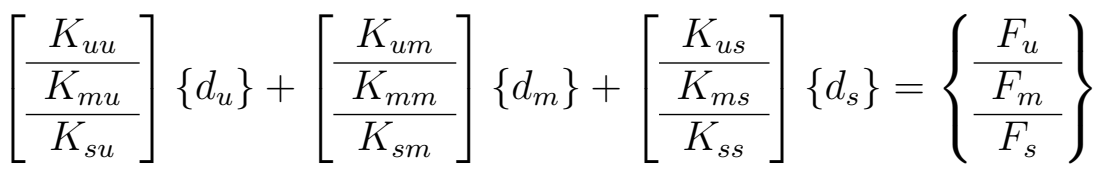

Writing the Eq. 48 in matrix form is obtained:

$$
\left\{d_{s}\right\}=\left[S_{s m}\right]\left\{d_{m}\right\}+\left\{\triangle_{d}\right\}
$$

Introducing Eq. A.3 in Eq. A.2.

$$
\left[\frac{K_{u u}}{\frac{K_{m u}}{K_{s u}}}\right]\left\{d_{u}\right\}+\left[\frac{K_{u m}}{\frac{K_{m m}}{K_{s m}}}\right]\left\{d_{m}\right\}+\left[\frac{K_{u s}}{\frac{K_{m s}}{K_{s s}}}\right]\left[S_{s m}\right]\left\{d_{m}\right\}+\left[\frac{K_{u s}}{\frac{K_{m s}}{K_{s s}}}\right]\left\{\triangle_{d}\right\}=\left\{\frac{F_{u}}{F_{m}} \frac{F_{s}}{F_{s}}\right\}
$$

and rewriting the equation in an orderly. 


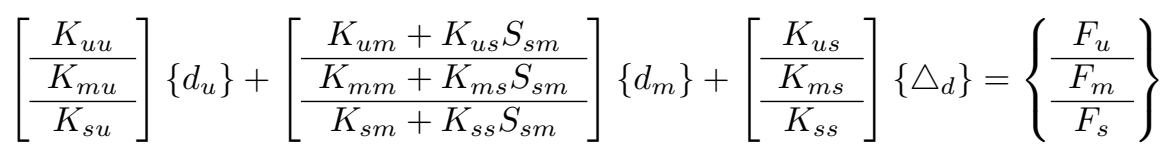

Writing the Eq. 49 in matrix form is obtained:

$$
\left\{F_{m}\right\}+\left[S_{m s}\right]\left\{F_{s}\right\}=0
$$

With Eq. A.6 it is possible to reduce the system of equations shown in Eq. A.5, which is:

$$
\begin{gathered}
{\left[\frac{K_{u u}}{K_{m u}+S_{m s} K_{s u}}\right]\left\{d_{u}\right\}+} \\
+\left[\frac{K_{u m}+K_{u s} S_{s m}}{K_{m m}+K_{m s} S_{s m}+S_{m s} K_{s m}+S_{m s} K_{s s} S_{s m}}\right]\left\{d_{m}\right\}+ \\
\quad+\left[\frac{K_{u s}}{K_{m s}+S_{m s} K_{s s}}\right]\left\{\triangle_{d}\right\}=\left\{\frac{F_{u}}{F_{m}+S_{m s} F_{s}}\right\}
\end{gathered}
$$

If the last equation is ordered and it is defined the reduced stiffness matrix, the reduced force vector and the reduced displacement vector as:

$$
\begin{gathered}
{\left[K_{r}\right]=\left[\begin{array}{c}
K_{u u} \\
{\left[d_{m u}+S_{m s} K_{s u}\right.}
\end{array}=\left\{\frac{d_{u m}+K_{u s} S_{s m}}{d_{m}}\right\}\right.} \\
{\left[F_{r}\right]=\left\{\frac{F_{u}-K_{u s}\left\{\triangle_{d}\right\}}{\left(K_{m s}+S_{m s} K_{s s}\right)\left\{\triangle_{d}\right\}}\right\}}
\end{gathered}
$$

the original problem to solve (Eq. 50) is reduced to:

$$
\mathbf{K}_{r} \cdot \mathbf{d}_{r}=\mathbf{F}_{r}
$$

The Eq. A.11 shows that the degrees of freedom of the slave nodes are not included in the displacement vector. The new equation system has less degrees of freedom than the original one and it also satisfies automatically the boudary conditions. 


\section{References}

[1] E. J. Barbero, Introduction to composite materials design, 2nd Edition, Taylor \& Francis, 2011.

[2] K. Váradi, Z. Néder, K. Friedrich, Fe contact an thermal analysis of composite surfeces with different fibre orientations subjected to a sliding steel asperity, Finite Elements in Analysis and Design 40 (2004) 1475 1497.

[3] T. Goda, K. Váradi, K. Friedrich, Fe micro-models to study contact states, stresses and failure mechanisms in a polymer composite subjected to a sliding steel asperity, Wear 251 (2001) 1584 - 1590.

[4] H. Huang, R. Talreja, Numerical simulation of matrix micro-cracking in short fiber reinforced polymer composites: Initiation and propagation, Composite Science and Technology 66 (2006) 2743 - 2757.

[5] M. N. Yuan, Y. Q. Yang, C. Li, P. Y. Heng, L. Z. Li, Numerical analysis of the stress-strain distributions in the particle reinforced metal matrix composite sic/6064al, Materials and Design 38 (2012) 1 - 6.

[6] C. A. Truesdell, R. Toupin, The classical field theories, Vol. 3 of Handbuch der Physik, Springer-Verlag, Berlin, 1960, Ch. 1, pp. 226-793.

[7] A. Green, J. Adkins, A contribution to the theory of non-linear diffusion, Archive for rational mechanics and analysis 15 (1964) 235 - 246.

[8] M. Ortiz, E. Popov, A physical model for the inelasticity of concrete, Proceedings of the royal society A383 (1982) $101-125$.

[9] M. Ortiz, E. Popov, Plain concrete as a composite material, Mechanics of materials 12 (1982) 139 - 150.

[10] S. Oller, L. Neamtu, E. Oñate, Una generalización de la teoría de mezclas clásica para el tratamiento de compuestos en serie/paralelo, in: Congreso Nacional de Materiales Compuestos, 1995, pp. 433 - 438.

[11] L. Neamtu, S. Oller, E. Oñate, A generalized mixing theory elastodamage-plastic model for finite element analysis of composites, in: Computational plasticity, 1997, pp. $1214-1219$. 
[12] S. Oller, Simulación numérica del comportamiento mecánico de los materiales compuestos, Centro Internacional de Métodos Numéricos en Ingeniería - CIMNE, 2003.

[13] F. Rastellini, S. Oller, O. Salomón, E. Oñate, Composite materials non-linear modelling for long fibre-reinforced laminates continuum basis, computational aspect and validations, Computers and Structures 86 (2008) 879-896.

[14] F. Otero, X. Martínez, S. Oller, O. Salomón, Study and prediction of the mechanical performance of a nanotube-reinforced composite, Composites Structures 94 (2012) 2920 - 2930.

[15] E. Sanchez-Palencia, Non-homogeneous media and vibration theory, in: Lecture Notes in Physics, Vol. 127, Springer-Verlag, Berlin, 1980.

[16] A. Bensoussan, J. Lions, G. Papanicolaou, Asymptotic Analysis for Periodic Structures, North-Holland, Amsterdam, 1978.

[17] P. M. Suquet, Plasticié et homogénésation (in french), Ph.D. thesis, Univ. Pierre et Marie Curie, Paris 6 (1982).

[18] P. M. Suquet, Elements of homogenizations for inelastic solid mechanics, in: Homogenization techniques for composite media, Springer, Berlin, 1987, pp. $193-279$.

[19] P. Suquet, Local and global aspects in the mathematical theory of plasticity, in: A. Sawczuk, G. Bianchi (Eds.), Plasticity Today - Modeling Methods and Applications, Elsevier, London, 1985.

[20] H. Moulinec, P. Suquet, A numerical method for computing the overall response of nonlinear composites with complex microstructure, Comput. Methods Appl. Mech. Engrg. 157 (1998) 69 - 94.

[21] C. Miehe, J. Schotte, J. Schroder, Computational micro-macro transitions and overall moduli in the analysis of polycrystals at large strains, Computational Materials Science 6 (1999) 372 - 382.

[22] C. Miehe, A. Koch, Computational micro-macro transitions of discretized microstructures undergoing small strains, Archive of Applied Mechanics 72 (2002) $300-317$. 
[23] C. Miehe, J. Shotte, M. Lambrecht, Homogenization of inelastic solid materials at finite strains based on incremental minimization principles. application to the texture analysis of polycrystals, Journal of the Mechanics and Physics of Solids 50 (10) (2002) 2123 - 2167.

[24] K. Terada, M. Hori, T. Kyoya, N. Kikuchi, Simulation of the multi-scale convergence in computational homogenization approaches, International Journal of Solids and Structures 37 (16) (2000) 2285 - 2311.

[25] K. Terada, N. Kikuchi, A class of general algorithms for multi-scale analyses of heterogeneous media, Comput. Methods Appl. Mech. Engrg. $190(40-41)(2001) 5247-5464$.

[26] M. G. D. Geers, V. G. Kouznetsova, W. A. M. Brekelmans, Gradientenhanced computational homogenization for the micro-macro scale transition, Journal de Physique IV 11 (5) (2001) 145 - 152.

[27] V. G. Kouznetsova, M. G. D. Geers, W. A. M. Brekelmans, Multiscale constitutive modelling of heterogeneous materials with a gradientenhanced computational homogenization scheme, Int. J. Numer. Meth. Engng 54 (2002) 1235 - 1260.

[28] V. G. Kouznetsova, M. G. D. Geers, W. A. M. Brekelmans, Multi-scale second-order computational homogenization of multi-phase materials: a nested finite element solution strategy, Comput. Methods. Appl. Mech. Engrg. 193 (48-51) (2004) 5525 - 5550.

[29] T. Massart, R. Peerlings, M. Geers, Structural damage analysis of masonry walls using computational homogenisation, International Journal of Damage Mecanics 16 (2007) 199 - 226.

[30] I. Ozdemir, W. A. M. Brekelmans, M. Geers, Fe2 computational homogenization for the thermo-mechanical analysis of heterogeneous solids, Comput. Methods. Appl. Mech. Engrg. 198 (2008) 602 - 613.

[31] K. Matous, M. G. Kulkarni, P. Geubelle, Multiscale cohesive failure modeling of heterogeneous adhesives, Journal of the Mechanics and Physics of Solids 56 (2008) 1511 - 1533.

[32] E. Car, S. Oller, E. Oñate, An anisotropic elastoplastic constitutive model for large strain analysis of fiber reinforced composite materials, 
Computer Methods in Applied Mechanics and Engineering 185 (2-4) (2000) 245-277.

[33] S. Oller, Numerical simulation of mechanical behavior of composite materials, 1st Edition, CIMNE-Springer, 2014.

[34] P. Mata, S. Oller, A. H. Barbat, Static analysis of beam structures under nonlinear geometric and constitutive behavior, Computer methods in applied mechanics and engineering 196.

[35] X. Martínez, S. Oller, F. Rastellini, A. H. Barbat, A numerical procedure simulating rc structures reinforced with frp using the serial/parallel mixing theory, Computers and Structures 86 (2008) 1604-1618.

[36] X. Martínez, S. Oller, E. Barbero, Study of delamination in composite by using the serial/parallel mixing theory and a damage formulation, Mechanical response of composites, Springer, 2008, pp. 119-140.

[37] X. Martínez, S. Oller, Numerical simulation of matrix reinforced composite materials subjected to compression loads, Archives of computational methods in engineering 16 (4) (2009) 357-397.

[38] J. A. Paredes, A. H. Barbat, S. Oller, A compression-tension concrete damage model, applied to a wind turbine reinforced concrete tower, Engineering Structures 33 (2011) 3559-3569.

[39] X. Martínez, F. Rastellini, S. Oller, F. Flores, E. Oñate, Computationally optimized formulation for the simulation of composite materials and delamination failures, Composites Part B: Engineering 42 (2011) 134144.

[40] M. A. Pérez, X. Martínez, S. Oller, L. Gil, F. Rastellini, F. Flores, Impact damage prediction in carbon fiber-reinforced laminated composite using the matrix-reinforced mixing theory, Composite Structures 104 (2013) 239-248.

[41] F. Zalamea, Tratamiento numérico de materiales compuestos mediante la teoría de homogeneización(in spanish), Ph.D. thesis, Strength of materials and structures in engineering department. Technical University of Catalonia (2000). 
[42] S. Oller, J. M. Canet, F. Zalamea, Composite material behavior using a homogenization double scale method, Journal of Engineering Mechanics 131 (1) (2005) $65-79$.

[43] H. Badillo, S. Oller, Numerical simulation of composite materials and structures using the unit cell homogenization approach, in: V International Conference on Science and Technology of Composite Materials, 2009.

[44] S. Giusti, P. Blanco, E. de Souza Neto, R. Feijóo, An assessment of the gurson yield criterion by a computational multi-scale approach, Engineering computations: International journal for computer-aided engineering and software 26 (3) (2009) $281-301$.

[45] J. Michel, H. Moulinec, P. Suquet, Effective properties of composite materials with periodic microstructure: a computational approach, Computer Methods in Applied Mechanics and Engineering 172 (1 - 4) (1999) $109-143$.

[46] E. de Souza Neto, R. Feijóo, Variational foundations of multi-scale constitutive models of solid: small and large kinematical formulation, in: LNCC Reseach and development report, no. 16, National Laboratory for Scientific Computing, Petrópolis, 2006.

[47] F. Devries, H. Dumontet, G. Duvaut, F. Lene, Homogenization and damage for composite structures, Int. J. Numer. Meth. Engng. 27 (2) (1989) $285-298$.

[48] S. Ghosh, K. Lee, S. Moorthy, Two scale analysis of heterogeneous elastic-plastic materials with asymptotic homogenization and voronoi cell finite element model, Computer Methods in Applied Mechanics and Engineering 132 (1 - 2) (1996) 63 - 116. 